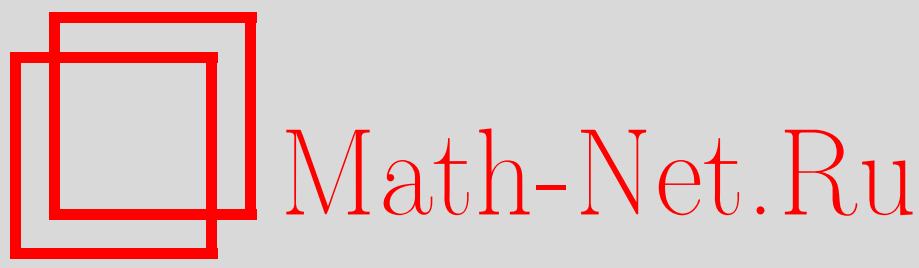

В. И. Хомич, О свойстве простой подстановки для суперинтуиционистских пропозициональных логик и о его связи с их свойством отделимости, Изв. РАН. Сер. матем., 2003, том 67, выпуск 2, 181-210

DOI: https://doi.org/10.4213/im431

Использование Общероссийского математического портала Math-Net.Ru подразумевает, что вы прочитали и согласны с пользовательским соглашением

http://www. mathnet.ru/rus/agreement

Параметры загрузки:

IP : 54.80 .73 .141

26 апреля 2023 г., 17:55:11 
УДК 510.64

\author{
В.И. Хомич
}

\title{
О свойстве простой подстановки для суперинтуиционистских пропозициональных логик и о его связи с их свойством отделимости
}

\begin{abstract}
Изучается свойство простой подстановки, касающееся суперинтуиционистских пропозициональных исчислений, являющихся аксиоматизациями суперинтуиционистских пропозициональных логик. Для этих исчислений получен алгебраический критерий наличия свойства простой подстановки. С его помощью доказано, что многие логики, среди которых содержатся почти все логики, порожденные формулами от одной переменной, не обладают свойством простой подстановки. Получен ряд результатов, устанавливающих связь между отделимыми и обладающими свойством простой подстановки аксиоматизациями рассматриваемых логик.

Библиографиия: 33 наименования.
\end{abstract}

\section{Введение}

Настоящая статья посвящена изучению введенного в работе [1] свойства простой подстановки, касающегося аксиоматизаций суперинтуиционистских пропозициональных логик - расширений интуиционистской пропозициональной логики [2]. Кроме того, будем интересоваться его связью со свойством отделимости, введенным в работе [3] и касаюшимся также аксиоматизаций рассматриваемых логик. Аксиоматизациями этих логик будут пропозищиональные исчисления, содержащие интуиционистское пропозициональное исчисление [2] как подсистему и называемые суперинтуиционистскими пропозициональными исчислениями.

В основе свойства простой подстановки лежит правило простой подстановки, предписывающее подставлять в имеющуюся у нас пропозициональную формулу вместо ее переменных только пропозициональные переменные. По сути, это свойство сводит решение вопроса о выводимости пропозициональных формул в данном исчислении к вопросу о выводимости определенным образом устроенных с помощью правила простой подстановки формул в интуиционистском пропозициональном исчислении. Естественным образом оно распространяется [1] на суперинтуиционистские пропозициональные логики: логика обладает свойством простой подстановки, если им обладает некоторая ее аксиоматизация.

Суперинтуиционистская пропозициональная логика называется отделимой,если сушествует ее аксиоматизация, удовлетворяюшая следующим условиям:

1) каждая ее аксиома содержит не более двух логических знаков, причем если их два, то один из них - импликация;

Работа выполнена при финансовой поддержке Российского фонда фундаментальных исследований (грант № 01-01-00154).

(C) в.и. Хомич, 2003 
2) какова бы ни была формула этой логики, существует ее вывод в данной аксиоматизации, формулы которого не содержат логических знаков, отличных от импликации и знаков рассматриваемой формулы.

Свойство простой подстановки изучалось в работах [1], [4], [5]. В [1] доказано, что абсолютно противоречивая, классическая и порожденная формулой $\neg b \vee \neg \neg b$ пропозициональные логики обладают свойством простой подстановки. Там же рассматривались суперинтуиционистские пропозициональные исчисления с единственной дополнительной аксиомой, являющейся формулой последовательности из [6], задаюшей классы эквивалентностей в интуиционистском пропозициональном исчислении формул от одной переменной. Установлено, что все они, кроме исчислений, аксиоматизирующих упомянутые вьше три логики, не обладают свойством простой подстановки. Однако остается открытым вопрос: обладают ли этим свойством логики, порожденные формулами от одной переменной и отличные от этих трех логик?

В работе [4] доказано, что табличные логики (т. е. логики, имеющие точную конечную модель) обладают свойством простой подстановки, а класс суперинтуиционистских пропозициональных логик, обладающих им, замкнут относительно операций пересечения и объединения. В [5] показано, что среди логик конечных слоев, определенных в [7], табличные и только они обладают этим свойством.

Известно [8], что предтабличных логик (т. е. логик, являюшихся максимальными относительно включения в классе не табличных) ровно три. В работах [1], [5] установлено, что одна из них обладает свойством простой подстановки, а две другие не обладают.

В настоящей работе доказаны результаты, анонсированные в [9], [10]. Доказана теорема 1 из [9], дающая критерий наличия свойства простой подстановки для суперинтуиционистских пропозициональных исчислений. Тем самым найдена алгебраическая форма этого свойства. Доказаны теоремы 2 и 3 из [9], первая из которых утверждает, что логика (см. [11]), порожденная формулой

$$
(\neg b \supset(c \vee d)) \supset((\neg b \supset c) \vee(\neg b \supset d))
$$

не обладает свойством простой подстановки, а вторая - что всякая логика, содержашаяся в порожденной формулой $\neg \neg b \vee(\neg \neg b \supset b)$ логике и удовлетворяюшая некоторому условию, не обладает этим свойством. Из теоремы 3 , в частности, следует, что почти все логики, порожденные формулами от одной переменной, не обладают им. Тем самым дан отрищательньй ответ на поставленньй выше вопрос. Для доказательства этих теорем получен ряд новых результатов, касающихся псевдобулевых алгебр специального вида. Отметим, что упомянутые результаты представляют и самостоятельный интерес.

Согласно результатам работ [1], [4], [5] и [3], [12]-[18] существуют логики, обладаюшие свойствами отделимости и простой подстановки, логики, не обладающие этими свойствами, и логики, обладающие одним из них и не обладающие другим. Поэтому возникает вопрос: каким образом связаны между собой отделимые и обладающие свойством простой подстановки аксиоматизации суперинтуиционистских пропозициональных логик? В настоящей работе доказаны теорема 4 и следствие 2 из [9], частично дающие ответ на этот вопрос. 


\section{§1. Предварительные сведения}

Введем необходимые понятия и обозначения. Интуиционистское пропозициональное исчисление [2], [19], заданное десятью аксиомами и двумя правилами вывода: подстановкой и модус поненс (modus ponens), будем обозначать через $\mathbf{H}$, а суперинтуищионистское пропозищиональное исчисление, получающееся из $\mathbf{H}$ путем добавления в список аксиом $\mathbf{H}$ конечного множества пропозициональных формул $\mathfrak{X}$, называемых его дополнительными аксиомами, - через $\mathbf{H}+\mathfrak{X}$. Исчисления $\mathbf{H}+\mathfrak{X}$ и $\mathbf{H}+\mathfrak{Y}$ называются равнооблемны.ми, если множество формул, выводимых в $\mathbf{H}+\mathfrak{X}$, совпадает с множеством формул, вьводимых в $\mathbf{H}+\mathfrak{Y}$.

Пропозициональные переменные и формулы будем обозначать строчными и заглавными латинскими буквами (возможно, с индексами) соответственно. Будем говорить, что пропозициональная формула $Y$ построена из переменных $b_{1}, \ldots, b_{n}$, если в $Y$ не входит никакая пропозициональная переменная, отличная от $b_{1}, \ldots, b_{n}$. Таким образом, мы допускаем, что некоторые из переменных $b_{1}, \ldots, b_{n}$ могут не входить в $Y$. Запись $\mathbf{F}\left(c_{1}, \ldots, c_{n}\left|X_{1}, \ldots, X_{n}\right| Z\right)$ будет означать результат подстановки в пропозициональную формулу $Z$ вместо пропозициональных переменных $c_{1}, \ldots, c_{n}$ пропозициональных формул $X_{1}, \ldots, X_{n}$ соответственно. Заметим, что некоторые из переменных $c_{1}, \ldots, c_{n}$ (даже все) могут не входить в $Z$.

Пусть пропозициональная формула $Y$ построена из переменных $b_{1}, \ldots, b_{n}$; $d_{1}, \ldots, d_{m}$ - различные пропозициональные переменные; $f_{1}, \ldots, f_{k}$ - все различные отображения множества $\left\{b_{1}, \ldots, b_{n}\right\}$ во множество $\left\{d_{1}, \ldots, d_{m}\right\}$. Формулу

$$
\underset{i=1}{k} \mathbf{F}\left(b_{1}, \ldots, b_{n}\left|f_{i}\left(b_{1}\right), \ldots, f_{i}\left(b_{n}\right)\right| Y\right)
$$

условимся записывать в виде $Y\left[d_{1}, \ldots, d_{m}\right]$. Скажем, что исчисление $\mathbf{H}+\mathfrak{X}$, где $\mathfrak{X} \neq \varnothing$, обладает свойством простой подстановки [1], если какова бы ни была пропозициональная формула $Z$, построенная из переменных $d_{1}, \ldots, d_{m}$ и вьводимая в $\mathbf{H}+\mathfrak{X}$, то в $\mathbf{H}$ выводима формула

$$
\left(\underset{X \in \mathfrak{X}}{\&} X\left[d_{1}, \ldots, d_{m}\right]\right) \supset Z
$$

Пусть $\nu$ - какой-нибудь набор логических пропозищиональных знаков. Пропозищиональную формулу назовем $\nu$-формулой, если она не содержит логических знаков, отличных от знаков набора $\nu$; $\nu$-формула называется $\nu$-выводимой в $\mathbf{H}+\mathfrak{X}$, если в $\mathbf{H}+\mathfrak{X}$ существует ее вывод, каждая формула которого является $\nu$-формулой. Исчисление $\mathbf{H}+\mathfrak{X}$ называется нормальным [12], если каждая его аксиома является $\supset \neg$-формулой, или $\supset \vee$-формулой, или $\supset \&$-формулой. Исчисление $\mathbf{H}+\mathfrak{X}$ называется $\nu$-полным $[20]$, если всякая $\nu$-формула, выводимая в $\mathbf{H}+\mathfrak{X}$, будет $\nu \cup\{\supset\}$-выводимой в нем. Исчисление $\mathbf{H}+\mathfrak{X}$ называется отделимым.м (см. [3], [19], [2], [21], [20]), если Н + $\mathfrak{X}$ является нормальным и $\nu$-полньм при любом $\nu$.

Суперинтуиционистской пропозициональной логикой называется множество пропозициональных формул, содержащее все аксиомы Н и замкнутое относительно правила подстановки и правила модус поненс. Суперинтуиционистское пропозищиональное исчисление называется аксиоматизацией суперинтуиционистской 
пропозициональной логики, если в нем выводимы все формулы этой логики и только они. Суперинтуиционистская пропозициональная логика называется аксиоматизируемой (нормализуемой [12], отделимой [12]), если существует ее аксиоматизация (нормальная аксиоматизация, отделимая аксиоматизация соответственно). Будем говорить, что суперинтуищионистская пропозициональная логика обладает свойством простой подстановки [1], если им обладает некоторая ее аксиоматизация.

Пусть теперь набор $\nu$ содержит знак импликации. Напомним определение понятия $\nu$-алгебры, введенного в работе [19] для изучения фрагментов исчисления $\mathbf{H}$ и ставшего обобщением таких уже известных под другими названиями понятий, как импликативная полуструктура, импликативная структура и псевдобулева алгебpa [22], [23]. По сути дела, $\nu$-алгебра - это множество, на котором заданы операции псевдобулевой алгебры, определяемые набором $\nu$ и включающие операцию относительного псевдодополнения. Точнее говоря, множество $\Xi$ назовем $\nu$-алгеброй, если в нем указан выделенный элемент, обозначаемый через $\mathbf{1}$, и заданы соответствующие логическим знакам набора $\nu$ операции ${ }^{1}$, удовлетворяющие следующим условиям:

1) $(\forall \xi \in \Xi)(1 \supset \xi=\mathbf{1} \Longrightarrow \xi=\mathbf{1})$;

2) $(\forall \xi, \eta \in \Xi)(\xi \supset \eta=\mathbf{1} \wedge \eta \supset \xi=\mathbf{1} \Longrightarrow \xi=\eta)$;

3) для каждой $\nu$-аксиомы $X$ (т. е. аксиомы, являющейся $\nu$-формулой) исчисления $\mathbf{H}$ значение всякого выражения, получающегося из $X$ путем замены переменных $\nu$-формулы $X$ элементами $\Xi$, а логических знаков - соответствующими им операциями $\Xi$, равно выделенному элементу.

Определение $\nu$-алгебры можно найти также в работах [18], [24]. Если $\nu$ содержит все четыре логических пропозищиональных знака, то $\nu$-алгебра является псевдобулевой алгеброй.

Пусть $\Theta$ - какая-либо $\nu$-алгебра, а $\xi, \eta$ - какие-нибудь ее элементы. На $\Theta$ можно задать отношение частичного порядка, положив $\xi \leqslant \eta$ в том и только том случае, когда $\xi \supset \eta=\mathbf{1}$. Элемент $\xi$ назовем предиественником в $\Theta$ элемента $\eta$ (обозначение $\xi<\eta)$, если $\xi \leqslant \eta$ и $\xi \neq \eta$. Элемент $\xi$ назовем непосредственным предшественником в $\Theta$ элемента $\eta$, если $\xi<\eta$ и в $\Theta$ не существует такого элемента $\zeta$, что $\xi<\zeta$ и $\zeta<\eta$. Если у выделенного элемента $\nu$-алгебры $\Theta$ имеется единственный непосредственный предшественник, то $\Theta$ назовем гёделевой. Элементы $\xi$ и $\eta$ назовем несравнимыми в $\Theta$, если $\xi \supset \eta \neq \mathbf{1}$ и $\eta \supset \xi \neq \mathbf{1}$; в противном случае элементы $\xi$ и $\eta$ будем называть сравнимыми в $\Theta$. Элемент $\xi$ назовем неразложимым әлементом $\nu$-алгебры $\Theta[17],[25]$, если из соотношений $\sigma, \tau \in \Theta$, $\xi \supset \sigma=\mathbf{1}, \xi \supset \tau=\mathbf{1}$ и $\sigma \supset(\tau \supset \xi)=\mathbf{1}$ следует, что $\xi=\sigma$ или $\xi=\tau$; в противном случае элемент $\xi$ будем называть разложимылм в $\Theta$. Это определение распространяет понятие неразложимого в пересечение элемента в импликативных структурах [26], [22] на $\nu$-алгебры потому, что для импликативных структур равенства $\xi \supset \sigma=\mathbf{1}, \xi \supset \tau=\mathbf{1}$ и $\sigma \supset(\tau \supset \xi)=\mathbf{1}$ равносильны равенству $\xi=\sigma \& \tau$.

Как и в работе [17], множество всех неразложимых элементов $\nu$-алгебры $\Theta$ будем обозначать через $I(\Theta)$. Очевидно, что $\mathbf{1} \in I(\Theta)$. Согласно [17, лемма 2] и [25, лемма 1] если $\sigma \in \Theta$ и $\tau \in I(\Theta)$, то в $\Theta$ верно хотя бы одно из равенств $\sigma \supset \tau=\tau$ или

\footnotetext{
${ }^{1}$ Операции $\nu$-алгебр обозначаются так же, как и соответствующие им (интерпретируемые ими) логические пропозициональные связки.
} 
$\sigma \supset \tau=1$. Поэтому подмножество $I(\Theta)$ множества $\Theta$ является $\supset$-подалгеброй $\nu$-алгебры $\Theta$.

Пусть $\Phi$ - какое-либо подмножество $\nu$-алгебры $\Theta$, а $\xi, \eta, \zeta_{1}, \ldots, \zeta_{n}$ - какие-нибудь ее элементы. Элемент $\sigma$ множества $\Phi$ назовем его минимальным элементом, если в $\Theta$ из соотношений $\tau \in \Phi$ и $\tau \leqslant \sigma$ следует, что $\tau=\sigma$.

Введем следуюшие обозначения: множество всех минимальных элементов множества $\Phi$ обозначим через $\mathfrak{M}(\Phi)$, пересечение в $\Theta$ всех элементов множества $\Phi$ (если в $\Theta$ определена операция пересечения и $\Phi$ конечно и непусто) через $\bar{\Phi}$, множество $\{\zeta \mid \zeta \in \Theta, \xi \supset \zeta=\mathbf{1}, \eta \supset \zeta=\mathbf{1}\}$ - через $\mathfrak{k}(\Theta, \xi, \eta)$, терм $\zeta_{n} \supset\left(\ldots \supset\left(\zeta_{1} \supset \xi\right) \ldots\right)$ - через $\left\{\zeta_{1}, \ldots, \zeta_{n}\right\} \supset \xi$ и наименьший элемент $\nu$-алгебры $\Theta$ (если он имеется) - через 0.

Конечное непустое множество $\Xi$ элементов из $\Theta$ назовем разложением элемента $\xi$ в $\Theta$, если $\Xi \supset \xi=\mathbf{1}$ и для всякого $\zeta$ из $\Xi$ верно соотношение $\xi \supset \zeta=\mathbf{1}$. Пусть $\Psi-$ какое-либо разложение элемента $\xi$ в $\Theta$. Элемент $\tau$ разложения $\Psi$ назовем сократимым әлементом, если $\Psi \backslash\{\tau\} \neq \varnothing$ и $(\Psi \backslash\{\tau\}) \supset \xi=\mathbf{1}$. В противном случае $\tau$ будем называть несократимым әлементом разложения $\Psi$. Разложение $\Psi$ назовем несократимым, если каждый его элемент является несократимым; в противном случае разложение $\Psi$ будем называть сократимым. Если в $\nu$-алгебре $\Theta$ для $\xi$ существует несократимое разложение, состоящее из неразложимых элементов, то оно единственно. В случае конечной $\nu$-алгебры $\Theta$ такое разложение для $\xi$ осушествимо [26], [27], а поэтому, имея в виду единственность, в дальнейшем будем обозначать его через $r(\xi)$.

Пусть $\mu$ и $\nu$ - наборы логических пропозициональных знаков, удовлетворяющие условиям $\supset \in \mu$ и $\mu \subseteq \nu$, а $\Xi$ и $\Theta$ - соответственно $\mu$-алгебра и $\nu$-алгебра. Будем говорить, что $\mu$-алгебра $\Xi$ вложима в $\nu$-алгебру $\Theta$, если существует изоморфизм $\mu$-алгебры $\Xi$ на некоторую $\mu$-подалгебру $\nu$-алгебры $\Theta$; сам изоморфизм будем называть вложением $\mu$-алгебры $\Xi$ в $\nu$-алгебру $\Theta$.

Пусть теперь $\& \in \nu, \mu$-алгебра $\Xi$ конечна и $f$ - отображение множества $I(\Xi)$ в $\Theta$. Как и в [17], для $f$ построим отображение $f^{\&}$ множества $\Xi$ в $\Theta$, положив $f^{\&}(\zeta)=\overline{f(r(\zeta))}$.

Пусть $X$ - какая-нибудь пропозициональная формула, а $\Phi$ - какая-либо псевдобулева алгебра. Формула $X$ называется общезначимой в подмножестве $\Xi$ алгебры $\Phi$, если значение каждого выражения, получаюшегося из $X$ путем замены переменных формулы $X$ элементами $\Xi$, а логических знаков - соответствующими им операциями алгебры $\Phi$, равно ее выделенному элементу. Если $\Xi=\Phi$, то будем говорить, что формула $X$ общезначима в алгебре $\Phi$ [23]. Если $X$ не является обшезначимой в $\Phi$, то $X$ назьвается опровержимой в $\Phi$ [23]. Формула $X$ называется наследственно общезначимой в алгебре $\Phi$ [9], [10], если для всякого множества ее образующих $\Psi$ верно, что из общезначимости $X$ в подмножестве $\Psi$ алгебры $\Phi$ следует общезначимость $X$ в $\Phi$.

Множество пропозициональных формул, общезначимых в какой-либо конечной псевдобулевой алгебре, множество всех пропозициональных формул и множество пропозициональных формул, выводимых в исчислении $\mathbf{H}+\{X\}$, являются суперинтуиционистскими пропозициональными логиками, которые называют табличной, абсолютно противоречивой и порожденной формулой $X$ логиками соответственно. Логику, порожденную формулой $X$, будем обозначать через $\mathfrak{L}(X)$. Суперинтуиционистская пропозициональная логика называется предтабличной, 
если она не таблична, но всякая логика, являюшаяся ее собственным расширением, таблична.

Запись $Y \vdash Z$ ( $Y \vDash Z)$ будет означать, что формула $Z$ выводима в $\mathbf{H}$ из формулы $Y$ с помощью обоих правил (с помощью правила модус поненс и выводимых в $\mathbf{H}$ формул), а запись $\vdash Y$ - что формула $Y$ выводима в Н. Конъюнкцию всех формул конечного непустого множества $\mathfrak{X}$ пропозициональных формул будем обозначать через $\overline{\mathfrak{X}}$, а число элементов конечного множества $\Phi$ - через $|\Phi|$. Пропозициональные формулы $X_{n} \supset\left(\ldots \supset\left(X_{1} \supset Z\right) \supset \cdots\right)$ и $(X \supset Y) \&(Y \supset X)$ условимся записывать в виде $\left\{X_{1}, \ldots, X_{n}\right\} \supset Y$ и $X \equiv Y$ соответственно. Знак $\equiv$ будем называть логическим знаком әквивалентности.

Пусть $\Xi$ и $\Psi$ - какие-либо множества, $\Phi$ - подмножество множества $\Xi$, а $f$ какое-нибудь отображение множества $\Xi$ в $\Psi$. Тогда множество $\{f(\zeta) \mid \zeta \in \Phi\}$ будем обозначать через $f(\Phi)$.

\section{§2. Алгебраическая форма свойства простой подстановки}

В этом параграфе докажем анонсированную в [9] теорему 1 об эквивалентности свойств простой подстановки и наследственной общезначимости пропозициональной формулы в псевдобулевой алгебре. Она показывает, что рассматриваемые понятия хотя и отличаются своими формами, но выражают одну и ту же суть. С помощью теоремы 1 сравнительно легко получим некоторые результаты работы [1], тем самым покажем возможность и целесообразность ее применения.

Пусть $\mathfrak{X}$ - непустое конечное множество пропозициональных формул. Нетрудно проверить, что исчисления $\mathbf{H}+\mathfrak{X}$ и $\mathbf{H}+\{\overline{\mathfrak{X}}\}$ равнообъемны и исчисление $\mathbf{H}+\mathfrak{X}$ обладает свойством простой подстановки тогда и только тогда, когда им обладает исчисление $\mathbf{H}+\{\overline{\mathfrak{X}}\}$. Поэтому, изучая это свойство для суперинтуиционистских пропозициональных исчислений и логик, достаточно рассматривать исчисления только с одной дополнительной аксиомой.

ЛЕмма 1. Пусть $Y$ - пропозичиональная формула, построенная из переменных $b_{1}, \ldots, b_{n}$. Тогда если логика $\mathfrak{L}(Y)$ обладает свойством простой подстановки, то существуют такие пропозициональные формуль $X_{1}^{1}, \ldots, X_{n}^{1}, \ldots$ $\ldots, X_{1}^{l}, \ldots, X_{n}^{l}$, чтоо исчисление

$$
\mathbf{H}+\left\{\mathbf{F}\left(b_{1}, \ldots, b_{n}\left|X_{1}^{i}, \ldots, X_{n}^{i}\right| Y\right) \mid 1 \leqslant i \leqslant l\right\}
$$

является аксиоматизацией логики $\mathfrak{L}(Y)$ и обладает свойством простой подстановки.

ДокАЗАТЕЛЬСтво. Пусть пропозициональная формула $Y$ построена из переменных $b_{1}, \ldots, b_{n}$ и логика $\mathfrak{L}(Y)$ обладает свойством простой подстановки. Тогда существует аксиоматизация $\mathbf{H}+\{Z\}$ логики $\mathfrak{L}(Y)$, обладающая свойством простой подстановки. Пусть формула $Z$ построена из переменных $c_{1}, \ldots, c_{k}$. Так как $Y \vdash Z$, то существуют пропозищиональные формулы $X_{1}^{1}, \ldots, X_{n}^{1}, \ldots, X_{1}^{l}, \ldots, X_{n}^{l}$, построенные из переменных $c_{1}, \ldots, c_{k}$ и удовлетворяющие условию

$$
\vdash \overline{\left\{\mathbf{F}\left(b_{1}, \ldots, b_{n}\left|X_{1}^{i}, \ldots, X_{n}^{i}\right| Y\right) \mid 1 \leqslant i \leqslant l\right\}} \supset Z .
$$

Множество $\left\{\mathbf{F}\left(b_{1}, \ldots, b_{n}\left|X_{1}^{i}, \ldots, X_{n}^{i}\right| Y\right) \mid 1 \leqslant i \leqslant l\right\}$ обозначим буквой $\mathfrak{Y}$. Легко видеть, что исчисление $\mathbf{H}+\mathfrak{Y}$ является аксиоматизацией логики $\mathfrak{L}(Y)$. Покажем, 
что исчисление $\mathbf{H}+\{\overline{\mathfrak{Y}}\}$ обладает свойством простой подстановки, тем самым докажем, что им обладает и исчисление $\mathbf{H}+\mathfrak{Y}$.

Пусть пропозициональная формула $T$ построена из переменных $d_{1}, \ldots, d_{m}$ и выводима в $\mathbf{H}+\{\overline{\mathfrak{Y}}\}$. Тогда $T$ вьводима в $\mathbf{H}+\{Z\}$. Поскольку исчисление $\mathbf{H}+\{Z\}$ обладает свойством простой подстановки, то $\vdash Z\left[d_{1}, \ldots, d_{m}\right] \supset T$. Поскольку формулы $\overline{\mathfrak{Y}}$ и $Z$ построены из переменных $c_{1}, \ldots, c_{k}$ и $\vdash \overline{\mathfrak{Y}} \supset Z$, то для каждой подстановки $f$ переменных $d_{1}, \ldots, d_{m}$ вместо переменных $c_{1}, \ldots, c_{k}$ верна выводимость

$$
\vdash \mathbf{F}\left(c_{1}, \ldots, c_{k}\left|f\left(c_{1}\right), \ldots, f\left(c_{k}\right)\right| \overline{\mathfrak{Y}}\right) \supset \mathbf{F}\left(c_{1}, \ldots, c_{k}\left|f\left(c_{1}\right), \ldots, f\left(c_{k}\right)\right| Z\right) .
$$

Тогда $\vdash \overline{\mathfrak{Y}}\left[d_{1}, \ldots, d_{m}\right] \supset Z\left[d_{1}, \ldots, d_{m}\right]$, ипоэтому имеем $\vdash \overline{\mathfrak{Y}}\left[d_{1}, \ldots, d_{m}\right] \supset T$. Следовательно, исчисление $\mathbf{H}+\{\overline{\mathfrak{Y}}\}$ обладает свойством простой подстановки. Лемма доказана.

Пусть $\Theta$ - конечная гёделева псевдобулева алгебра, а $X$ - пропозициональная формула, построенная из переменных $b_{1}, \ldots, b_{n}$. Опровержение $g$ формулы $X$ в $\Theta$ называется предельным [28], если $g(X)=\omega$, где $\omega$ - единственный непосредственный предшественник выделенного элемента алгебры $\Theta$, и элементы $g\left(b_{1}\right), \ldots, g\left(b_{n}\right)$ порождают $\Theta$ с помошью ее операций. Если формула $X$ опровержима в $\Theta$ и каждое опровержение формулы $X$ в $\Theta$ является предельным, то формула $X$ называется предельно опровержимой в $\Theta[28]$.

Докажем анонсированную в работе [9] теорему 1.

Теорема 1 [9, теорема 1]. Какова бы ни бълла пропозициональная формула $Y$, исчисление $\mathbf{H}+\{Y\}$ обладает свойством простой подстановки тогда и только тогда, когда формула $Y$ наследственно общезначима в каждой конечной гёделевой псевдобулевой алгебре.

ДокАЗАТЕЛЬСТво. Пусть пропозициональная формула $Y$ построена из переменных $b_{1}, \ldots, b_{n}$. Пусть исчисление $\mathbf{H}+\{Y\}$ обладает свойством простой подстановки. Рассмотрим конечную гёделеву псевдобулеву алгебру $\Theta$. Покажем, что формула $Y$ наследственно общезначима в $\Theta$. Пусть $\xi_{1}, \ldots, \xi_{m}$ - образующие алгебры $\Theta$, и пусть формула $Y$ общезначима в множестве $\left\{\xi_{1}, \ldots, \xi_{m}\right\}$ алгебры $\Theta$. Зафиксируем оценку $f$ переменных $b_{1}, \ldots, b_{n}$ в $\Theta$. Для $f\left(b_{i}\right)$, где $1 \leqslant i \leqslant n$, существует такой терм $\mathscr{B}_{i}\left(\xi_{1}, \ldots, \xi_{m}\right)$, что $f\left(b_{i}\right)=\mathscr{B}_{i}\left(\xi_{1}, \ldots, \xi_{m}\right)$. Пусть $d_{1}, \ldots, d_{m}-$ различные пропозициональные переменные. Заменив в терме $\mathscr{B}_{i}\left(\xi_{1}, \ldots, \xi_{m}\right)$ элемент $\xi_{j}$ пропозициональной переменной $d_{j}$, а операции $\Theta$ - соответствующими им логическими пропозициональными связками, построим формулу $B_{i}\left(d_{1}, \ldots, d_{m}\right)$.

Положим $W=\mathbf{F}\left(b_{1}, \ldots, b_{n}\left|B_{1}, \ldots, B_{n}\right| Y\right)$. Легко видеть, что формула $W$ построена из переменных $d_{1}, \ldots, d_{m}$ и вьводима в $\mathbf{H}+\{Y\}$. Поскольку исчисление $\mathbf{H}+\{Y\}$ обладает свойством простой подстановки, то $\vdash Y\left[d_{1}, \ldots, d_{m}\right] \supset W$. Построим оценку $g$ переменных $d_{1}, \ldots, d_{m}$ в $\Theta$, положив $g\left(d_{j}\right)=\xi_{j}$, где $1 \leqslant j \leqslant m$. Тогда $g\left(Y\left[d_{1}, \ldots, d_{m}\right] \supset W\right)=\mathbf{1}$ и, следовательно, $g\left(Y\left[d_{1}, \ldots, d_{m}\right]\right) \supset g(W)=\mathbf{1}$. Поскольку $Y$ общезначима во множестве $\left\{\xi_{1}, \ldots, \xi_{m}\right\}$ алгебры $\Theta$, имеем $g\left(Y\left[d_{1}, \ldots, d_{m}\right]\right)=1$. Значит, $g(W)=1$. Так как $f\left(b_{i}\right)=g\left(B_{i}\right)$, где $1 \leqslant$ $i \leqslant n$, то $f(Y)=g(W)$, и поэтому имеем $f(Y)=\mathbf{1}$. Следовательно, формула $Y$ общезначима в $\Theta$. Таким образом, $Y$ наследственно общезначима в $\Theta$.

Пусть формула $Y$ наследственно обшезначима в каждой конечной гёделевой псевдобулевой алгебре. Покажем, что исчисление $\mathbf{H}+\{Y\}$ обладает свойством 
простой подстановки. Пусть формула $X$ построена из переменных $c_{1}, \ldots, c_{k}$ и выводима в $\mathbf{H}+\{Y\}$. Докажем, что $\vdash Y\left[c_{1}, \ldots, c_{k}\right] \supset X$. Предположим, что это не так. Тогда найдется [29], [30] конечная псевдобулева алгебра, в которой формула $R=Y\left[c_{1}, \ldots, c_{k}\right] \supset X$ опровержима. Поэтому согласно [28, лемма 5] существует конечная гёделева псевдобулева алгебра $\Xi$, в которой $R$ предельно опровержима. Следовательно, найдется такое опровержение $h$ формулы $R$ в $\Theta$, что $h(R)=\omega$, где $\omega$ - единственный непосредственный предшественник выделенного элемента алгебры $\Xi$, и элементы $h\left(c_{1}\right), \ldots, h\left(c_{k}\right)$ являются образуюшими алгебры $\Xi$. Поскольку $\Xi$ - гёделева, имеем $h\left(Y\left[c_{1}, \ldots, c_{k}\right]\right)=\mathbf{1}$ и $h(X)=\omega$. Поэтому формула $Y$ общезначима во множестве $\left\{h\left(c_{1}\right), \ldots, h\left(c_{k}\right)\right\}$ алгебры $\Xi$, а $X$ опровержима в $\Xi$. Так как $Y$ наследственно обшезначима в $\Xi$, то $Y$ общезначима в $\Xi$. Поскольку $X$ выводима в $\mathbf{H}+\{Y\}$, то $X$ обшезначима в $\Xi$. Противоречие. Следовательно, $\vdash Y\left[c_{1}, \ldots, c_{k}\right] \supset X$. Таким образом, исчисление $\mathbf{H}+\{Y\}$ обладает свойством простой подстановки. Теорема доказана.

Нетрудно проверить, что во всякой гёделевой псевдобулевой алгебре для любых ее элементов $\xi$ и $\eta$ выполнено следуюшее условие: если $\xi \vee \eta=\mathbf{1}$, то $\xi=\mathbf{1}$ или $\eta=1$. Этим фактом будем пользоваться далее.

Покажем, что с помощью теоремы 1 сравнительно легко получаются некоторые результаты работы [1], касающиеся свойства простой подстановки. Сначала докажем следующую лемму.

ЛЕмма 2. Пропозичиональные формуль $b \vee \neg b, \neg b \vee \neg \neg b \quad u(\neg b \vee \neg \neg b) \&$ $((c \supset d) \vee((c \supset d) \supset c))$ наследственно общезначимы во всякой конечной гёделевой псевдобулевой алгебре.

ДокАЗАтЕльство. Пусть задана конечная гёделева псевдобулева алгебра $\Theta$. Покажем, что формула $b \vee \neg b$ наследственно обшезначима в $\Theta$. Пусть $\Xi-$ множество образующих алгебры $\Theta$, и пусть для любого $\zeta$ из $\Xi$ в $\Theta$ верно равенство $\zeta \vee \neg \zeta=1$. Покажем, что формула $b \vee \neg b$ общезначима в $\Theta$. Для этого достаточно доказать, что для всякого терма $\mathscr{B}$, построенного из элементов множества с помошью операций алгебры $\Theta$, в $\Theta$ верно равенство $\mathscr{B} \vee \neg \mathscr{B}=\mathbf{1}$. Докажем это методом индукции по построению терма $\mathscr{B}$. Если $\mathscr{B}-$ элемент из $\Xi$, то $\mathscr{B} \vee \neg \mathscr{B}=\mathbf{1}$. Пусть $\mathscr{B}=\mathscr{C} \circ \mathscr{D}$, где $\mathscr{C}$ и $\mathscr{D}$ - термы, построенные из элементов множества $\Xi$ с помошью операций алгебры $\Theta$, а о - один из знаков $\supset$, или $\&$, или $\vee$. Пусть $\mathscr{C} \vee \neg \mathscr{C}=\mathbf{1}$ и $\mathscr{D} \vee \neg \mathscr{D}=\mathbf{1}$.

Пусть о есть $\supset$. Если $\mathscr{C}=\mathbf{0}$ или $\mathscr{D}=\mathbf{1}$, то $\mathscr{B}=\mathbf{1}$, и поэтому имеем $\mathscr{B} \vee \neg \mathscr{B}=\mathbf{1}$. Пусть $\mathscr{C} \neq \mathbf{0}$ и $\mathscr{D} \neq \mathbf{1}$. Тогда $\neg \mathscr{C} \neq \mathbf{1}$. Так как $\mathscr{C} \vee \neg \mathscr{C}=\mathbf{1}, \mathscr{D} \vee \neg \mathscr{D}=\mathbf{1}$ и $\Theta-$ гёделева алгебра, то $\mathscr{C}=\mathbf{1}$ и $\neg \mathscr{D}=\mathbf{1}$. Значит, $\mathscr{D}=\mathbf{0}$. Тогда $\mathscr{B}=\mathbf{0}$, и поэтому имеем $\neg \mathscr{B}=\mathbf{1}$. Следовательно, $\mathscr{B} \vee \neg \mathscr{B}=\mathbf{1}$.

Пусть о есть \&. Если $\neg \mathscr{C}=\mathbf{1}$ или $\neg \mathscr{D}=\mathbf{1}$, то $\mathscr{C}=\mathbf{0}$ или $\mathscr{D}=\mathbf{0}$, а следовательно, $\mathscr{B}=\mathbf{0}$, и тем самым имеем $\mathscr{B} \vee \neg \mathscr{B}=\mathbf{1}$. Пусть $\neg \mathscr{C} \neq \mathbf{1}$ и $\neg \mathscr{D} \neq \mathbf{1}$. Так как $\mathscr{C} \vee \neg \mathscr{C}=\mathbf{1}, \mathscr{D} \vee \neg \mathscr{D}=\mathbf{1}$ и $\Theta-$ гёделева алгебра, то $\mathscr{C}=\mathbf{1}$ и $\mathscr{D}=\mathbf{1}$. Тогда $\mathscr{B}=\mathbf{1}$, и поэтому имеем $\mathscr{B} \vee \neg \mathscr{B}=\mathbf{1}$.

Пусть о есть $\vee$. Если $\mathscr{C}=\mathbf{1}$ или $\mathscr{D}=\mathbf{1}$, то $\mathscr{B}=\mathbf{1}$ и, следовательно, $\mathscr{B} \vee \neg \mathscr{B}=\mathbf{1}$. Пусть $\mathscr{C} \neq \mathbf{1}$ и $\mathscr{D} \neq \mathbf{1}$. Поскольку $\mathscr{C} \vee \neg \mathscr{C}=\mathbf{1}, \mathscr{D} \vee \neg \mathscr{D}=\mathbf{1}$ и $\Theta$ - гёделева алгебра, то $\neg \mathscr{C}=\mathbf{1}$ и $\neg \mathscr{D}=\mathbf{1}$. Значит, $\mathscr{C}=\mathbf{0}$ и $\mathscr{D}=\mathbf{0}$. Тогда $\mathscr{B}=\mathbf{0}$, и поэтому имеем $\mathscr{B} \vee \neg \mathscr{B}=\mathbf{1}$. 
Пусть $\mathscr{B}=\neg \mathscr{C}$, где $\mathscr{C}$ - терм, построенный из элементов множества $\Xi$ с помощюю операций алгебры $\Theta$. Пусть $\mathscr{C} \vee \neg \mathscr{C}=\mathbf{1}$. Если $\mathscr{B}=\mathbf{1}$, то имеем $\mathscr{B} \vee \neg \mathscr{B}=\mathbf{1}$. Пусть $\mathscr{B} \neq \mathbf{1}$, т.е. $\neg \mathscr{C} \neq \mathbf{1}$. Поскольку $\mathscr{C} \vee \neg \mathscr{C}=\mathbf{1}$ и $\Theta-$ гёделева алгебра, то $\mathscr{C}=\mathbf{1}$. Значит, $\neg \neg \mathscr{C}=\mathbf{1}$, т. е. $\neg \mathscr{B}=\mathbf{1}$. Следовательно, $\mathscr{B} \vee \neg \mathscr{B}=\mathbf{1}$.

Покажем, что формула $\neg b \vee \neg \neg b$ наследственно общезначима в $\Theta$. Пусть $\Xi-$ множество образуюших алгебры $\Theta$, и пусть для любого $\zeta$ из $\Xi$ в $\Theta$ верно равенство $\neg \zeta \vee \neg \neg \zeta=\mathbf{1}$. Покажем, что формула $\neg b \vee \neg \neg b$ обшезначима в $\Theta$. Для этого достаточно доказать, что для всякого терма $\mathscr{B}$, построенного из элементов множества $\Xi$ с помощью операций алгебры $\Theta$, в $\Theta$ верно равенство $\neg \mathscr{B} \vee \neg \neg \mathscr{B}=1$. Докажем это методом индукции по построению терма $\mathscr{B}$. Если $\mathscr{B}$ - элемент из $\Xi$, то $\mathscr{B} \vee \neg \mathscr{B}=\mathbf{1}$. Пусть $\mathscr{B}=\mathscr{C}$ ○ $\mathscr{D}$, где $\mathscr{C}$ и $\mathscr{D}$ - термы, построенные из элементов множества $\Xi$ с помощью операций алгебры $\Theta$, а о - один из знаков $\supset$, или \&, или $\vee$. Пусть $\neg \mathscr{C} \vee \neg \neg \mathscr{C}=\mathbf{1}$ и $\neg \mathscr{D} \vee \neg \neg \mathscr{D}=\mathbf{1}$

Пусть $\circ$ есть $\supset$. Если $\neg \mathscr{C}=\mathbf{1}$ или $\neg \neg \mathscr{D}=\mathbf{1}$, то $\neg \neg \mathscr{B}=\neg \neg(\mathscr{C} \supset \mathscr{D})=$ $\neg \neg \mathscr{C} \supset \neg \neg \mathscr{D}=\neg \mathbf{1} \supset \neg \neg \mathscr{D}=\mathbf{1}$ или $\neg \neg \mathscr{B}=\neg \neg \mathscr{C} \supset \neg \neg \mathscr{D}=\neg \neg \mathscr{C} \supset \mathbf{1}=\mathbf{1}$, а следовательно, $\neg \mathscr{B} \vee \neg \neg \mathscr{B}=\mathbf{1}$. Пусть $\neg \mathscr{C} \neq \mathbf{1}$ и $\neg \neg \mathscr{D} \neq \mathbf{1}$. Поскольку $\neg \mathscr{C} \vee \neg \neg \mathscr{C}=\mathbf{1}$, $\neg \mathscr{D} \vee \neg \neg \mathscr{D}=\mathbf{1}$ и $\Theta-$ гёделева алгебра, то $\neg \neg \mathscr{C}=\mathbf{1}$ и $\neg \mathscr{D}=\mathbf{1}$. Тогда

$$
\neg \mathscr{B}=\neg \neg \neg \mathscr{B}=\neg \neg \neg(\mathscr{C} \supset \mathscr{D})=\neg(\neg \neg \mathscr{C} \supset \neg \neg \mathscr{D})=\neg(\mathbf{1} \supset \neg \mathbf{1})=\mathbf{1},
$$

и поэтому имеем $\neg \mathscr{B} \vee \neg \neg \mathscr{B}=\mathbf{1}$.

Пусть $\circ$ есть \&. Пусть $\neg \mathscr{C}=\mathbf{1}$ или $\neg \mathscr{D}=\mathbf{1}$. Тогда $\neg \neg \mathscr{C}=\mathbf{0}$ или $\neg \neg \mathscr{D}=\mathbf{0}$. Поэтому имеем

$$
\neg \mathscr{B}=\neg \neg \neg \mathscr{B}=\neg \neg \neg(\mathscr{C} \& \mathscr{D})=\neg(\neg \neg \mathscr{C} \& \neg \neg \mathscr{D})=\neg(\mathbf{0} \& \neg \neg \mathscr{D})=\mathbf{1}
$$

или

$$
\neg \mathscr{B}=\neg(\neg \neg \mathscr{C} \& \neg \neg \mathscr{D})=\neg(\neg \neg \mathscr{C} \& \mathbf{0})=\mathbf{1} .
$$

Следовательно, $\neg \mathscr{B} \vee \neg \neg \mathscr{B}=\mathbf{1}$. Пусть $\neg \mathscr{C} \neq \mathbf{1}$ и $\neg \mathscr{D} \neq \mathbf{1}$. Так как $\neg \mathscr{C} \vee \neg \neg \mathscr{C}=\mathbf{1}$, $\neg \mathscr{D} \vee \neg \neg \mathscr{D}=\mathbf{1}$ и $\Theta-$ гёделева алгебра, то $\neg \neg \mathscr{C}=\mathbf{1}$ и $\neg \neg \mathscr{D}=\mathbf{1}$. Тогда $\neg \neg \mathscr{B}=$ $\neg \neg(\mathscr{C} \& \mathscr{D})=\neg \neg \mathscr{C} \& \neg \neg \mathscr{D}=\mathbf{1}$, и поэтому имеем $\neg \mathscr{B} \vee \neg \neg \mathscr{B}=\mathbf{1}$

Пусть о есть $\vee$. Пусть $\neg \neg \mathscr{C}=\mathbf{1}$ или $\neg \neg \mathscr{D}=\mathbf{1}$. Тогда $\neg \mathscr{C}=\neg \neg \neg \mathscr{C}=\mathbf{0}$ или $\neg \mathscr{D}=\neg \neg \neg \mathscr{D}=\mathbf{0}$. Следовательно,

$$
\neg \neg \mathscr{B}=\neg \neg(\mathscr{C} \vee \mathscr{D})=\neg(\neg \mathscr{C} \& \neg \mathscr{D})=\neg(\mathbf{0} \& \neg \mathscr{D})=\mathbf{1}
$$

или

$$
\neg \neg \mathscr{B}=\neg(\neg \mathscr{C} \& \neg \mathscr{D})=\neg(\neg \mathscr{C} \& \mathbf{0})=\mathbf{1} .
$$

Значит, $\neg \mathscr{B} \vee \neg \neg \mathscr{B}=1$. Пусть $\neg \neg \mathscr{C} \neq 1$ и $\neg \neg \mathscr{D} \neq \mathbf{1}$. Так как $\neg \mathscr{C} \vee \neg \neg \mathscr{C}=\mathbf{1}$, $\neg \mathscr{D} \vee \neg \neg \mathscr{D}=\mathbf{1}$ и $\Theta-$ гёделева алгебра, то $\neg \mathscr{C}=\mathbf{1}$ и $\neg \mathscr{D}=\mathbf{1}$. Тогда $\neg \mathscr{B}=$ $\neg(\mathscr{C} \vee \mathscr{D})=\neg \mathscr{C} \& \neg \mathscr{D}=\mathbf{1}$, и поэтому имеем $\neg \mathscr{B} \vee \neg \neg \mathscr{B}=\mathbf{1}$

Пусть $\mathscr{B}=\neg \mathscr{C}$, где $\mathscr{C}$ - терм, построенный из элементов множества $\Xi$ с помощью операций алгебры $\Theta$. Пусть $\neg \mathscr{C} \vee \neg \neg \mathscr{C}=\mathbf{1}$. Следовательно,

$$
\neg \mathscr{B} \vee \neg \neg \mathscr{B}=\neg \neg \mathscr{C} \vee \neg \neg \neg \mathscr{C}=\neg \neg \mathscr{C} \vee \neg \mathscr{C}=\mathbf{1}
$$


Покажем, что формула $(\neg b \vee \neg \neg b) \&((c \supset d) \vee((c \supset d) \supset c))$ наследственно общезначима в $\Theta$. Пусть $\Xi$ - множество образующих алгебры $\Theta$, и пусть для любых $\zeta$, $\xi$ и $\eta$ из $\Xi$ в $\Theta$ верно равенство

$$
(\neg \zeta \vee \neg \neg \zeta) \&((\xi \supset \eta) \vee((\xi \supset \eta) \supset \xi))=\mathbf{1}
$$

и тем самым верны равенства $\neg \zeta \vee \neg \neg \zeta=1$ и $(\xi \supset \eta) \vee((\xi \supset \eta) \supset \xi)=\mathbf{1}$. Покажем, что формула $(\neg b \vee \neg \neg b) \&((c \supset d) \vee((c \supset d) \supset c))$ общезначима в $\Theta$. Для этого достаточно доказать, что для любых термов $\mathscr{B}, \mathscr{F}$ и $\mathscr{G}$, построенных из элементов множества $\Xi$ с помощью операций алгебры $\Theta$, в $\Theta$ верны равенства $\neg \mathscr{B} \vee \neg \neg \mathscr{B}=\mathbf{1}$ и $(\mathscr{F} \supset \mathscr{G}) \vee((\mathscr{F} \supset \mathscr{G}) \supset \mathscr{F})=\mathbf{1}$. В силу доказанного выше первое равенство имеет место. Докажем второе. Если $\mathscr{F}=\mathbf{1}$ или $\mathscr{G}=\mathbf{1}$, то имеем $(\mathscr{F} \supset \mathscr{G}) \vee((\mathscr{F} \supset \mathscr{G}) \supset \mathscr{F})=\mathbf{1}$. Пусть $\mathscr{F} \neq \mathbf{1}$ и $\mathscr{G} \neq \mathbf{1}$. Индукцией по числу вхождений операций алгебры $\Theta$ в термы $\mathscr{F}$ и $\mathscr{G}$ докажем, что $\mathscr{R}=$ $(\mathscr{F} \supset \mathscr{G}) \vee((\mathscr{F} \supset \mathscr{G}) \supset \mathscr{F})=\mathbf{1}$. Если $\mathscr{F}$ и $\mathscr{G}$ - элементы из $\Xi$, то $\mathscr{R}=\mathbf{1}$.

Пусть $\mathscr{F}$ - указанный терм, а $\mathscr{G}=\neg \mathscr{S}$, где $\mathscr{S}$ - терм, построенный из элементов множества $\Xi$ с помошью операций алгебры $\Theta$. В силу доказанного выше имеем $\neg \mathscr{S} \vee \neg \neg \mathscr{S}=\mathbf{1}$ и $\neg \mathscr{F} \vee \neg \neg \mathscr{F}=\mathbf{1}$. Так как $\mathscr{G} \neq \mathbf{1}$ (т.е. $\neg \mathscr{S} \neq \mathbf{1}$ ) и $\Theta$ - гёделева алгебра, то $\neg \neg \mathscr{S}=\mathbf{1}$. Следовательно, $\mathscr{G}=\neg \mathscr{S}=\neg \neg \neg \mathscr{S}=\mathbf{0}$. Значит,

$$
\mathscr{R}=(\mathscr{F} \supset \mathbf{0}) \vee((\mathscr{F} \supset \mathbf{0}) \supset \mathscr{F})=\neg \mathscr{F} \vee(\neg \mathscr{F} \supset \mathscr{F})=\neg \mathscr{F} \vee \neg \neg \mathscr{F}=\mathbf{1} .
$$

Пусть $\mathscr{G}$ - указанный терм, а $\mathscr{F}=\neg \mathscr{P}$, где $\mathscr{P}$ - терм, построенный из элементов множества $\Xi$ с помощью операций алгебры $\Theta$. В силу доказанного выше имеем $\neg \mathscr{P} \vee \neg \neg \mathscr{P}=\mathbf{1}$. Так как $\mathscr{F} \neq \mathbf{1}$ (т. е. $\neg \mathscr{P} \neq \mathbf{1})$ и $\Theta-$ гёделева алгебра, то $\neg \neg \mathscr{P}=\mathbf{1}$. Следовательно, $\mathscr{F}=\neg \mathscr{P}=\neg \neg \neg \mathscr{P}=\mathbf{0}$. Значит,

$$
\mathscr{R}=(\mathbf{0} \supset \mathscr{G}) \vee((\mathbf{0} \supset \mathscr{G}) \supset \mathbf{0})=\mathbf{1} \vee(\mathbf{1} \supset \mathbf{0})=\mathbf{1} .
$$

Пусть $\mathscr{F}=\mathscr{P} \circ \mathscr{Q}$ и $\mathscr{G}=\mathscr{S} * \mathscr{T}$, где $\mathscr{P}, \mathscr{Q}, \mathscr{S}$ и $\mathscr{T}$ - термы, построенные из элементов множества $\Xi$ с помощью операций алгебры $\Theta$, а о и $*$ - какие-нибудь из знаков $\supset$, или $\&$, или $\vee$. Пусть для любых термов $\mathscr{X}$ и $\mathscr{Y}$ из множества $\{\mathscr{P}, \mathscr{Q}, \mathscr{S}, \mathscr{T}\}$ в $\Theta$ верно равенство $(\mathscr{X} \supset \mathscr{Y}) \vee((\mathscr{X} \supset \mathscr{Y}) \supset \mathscr{X})=\mathbf{1}$.

Пусть $\circ$ и * суть $\supset$. Поскольку $\mathscr{F} \neq \mathbf{1}$ (т.е. $\mathscr{P} \supset \mathscr{Q} \neq \mathbf{1}),(\mathscr{P} \supset \mathscr{Q}) \vee((\mathscr{P} \supset$ $\mathscr{Q}) \supset \mathscr{P})=1, \quad \mathscr{G} \neq \mathbf{1}$ (т.е. $\mathscr{S} \supset \mathscr{T} \neq \mathbf{1}), \quad(\mathscr{S} \supset \mathscr{T}) \vee((\mathscr{S} \supset \mathscr{T}) \supset \mathscr{S})=1$ и $\Theta$ - гёделева алгебра, то $(\mathscr{P} \supset \mathscr{Q}) \supset \mathscr{P}=\mathbf{1}$ и $(\mathscr{S} \supset \mathscr{T}) \supset \mathscr{S}=\mathbf{1}$. Поскольку $((\mathscr{P} \supset \mathscr{Q}) \supset \mathscr{P}) \supset((\mathscr{P} \supset \mathscr{Q}) \supset \mathscr{Q})=\mathbf{1}$ и $((\mathscr{S} \supset \mathscr{T}) \supset \mathscr{S}) \supset((\mathscr{S} \supset \mathscr{T}) \supset \mathscr{T})=\mathbf{1}$, то имеем $(\mathscr{P} \supset \mathscr{Q}) \supset \mathscr{Q}=\mathbf{1}$ и $(\mathscr{S} \supset \mathscr{T}) \supset \mathscr{T}=\mathbf{1}$. Легко видеть, что $\mathscr{Q} \supset(\mathscr{P} \supset \mathscr{Q})=\mathbf{1}$ и $\mathscr{T} \supset(\mathscr{S} \supset \mathscr{T})=1$. Значит, $\mathscr{F}=\mathscr{P} \supset \mathscr{Q}=\mathscr{Q}$ и $\mathscr{G}=\mathscr{S} \supset \mathscr{T}=\mathscr{T}$. Следовательно,

$$
\mathscr{R}=(\mathscr{Q} \supset \mathscr{T}) \vee((\mathscr{Q} \supset \mathscr{T}) \supset \mathscr{Q})=\mathbf{1} .
$$

Пусть $\circ$ есть $\supset$, а $*$ есть $\&$. Так как $\mathscr{F} \neq \mathbf{1}$ (т.е. $\mathscr{P} \supset \mathscr{Q} \neq \mathbf{1}$ ), то, как и в предыдушем случае, получаем, что $\mathscr{F}=\mathscr{Q}$. Если $\mathscr{S} \supset \mathscr{T}=\mathbf{1}$, то $\mathscr{G}=\mathscr{S} \& \mathscr{T}=\mathscr{S}$, и поэтому имеем $\mathscr{R}=(\mathscr{Q} \supset \mathscr{S}) \vee((\mathscr{Q} \supset \mathscr{S}) \supset \mathscr{Q})=1$. Пусть $\mathscr{S} \supset \mathscr{T} \neq 1$. Так как $(\mathscr{S} \supset \mathscr{T}) \vee((\mathscr{S} \supset \mathscr{T}) \supset \mathscr{S})=\mathbf{1}$ и $\Theta$ - гёделева алгебра, то $(\mathscr{S} \supset \mathscr{T}) \supset \mathscr{S}=\mathbf{1}$. Поскольку $((\mathscr{S} \supset \mathscr{T}) \supset \mathscr{S}) \supset(\mathscr{T} \supset \mathscr{S})=\mathbf{1}$, то имеем $\mathscr{T} \supset \mathscr{S}=\mathbf{1}$. Значит, $\mathscr{G}=$ $\mathscr{S} \& \mathscr{T}=\mathscr{T}$. Следовательно,

$$
\mathscr{R}=(\mathscr{Q} \supset \mathscr{T}) \vee((\mathscr{Q} \supset \mathscr{T}) \supset \mathscr{Q})=\mathbf{1} .
$$


Пусть ○ есть $\supset$, а $*$ есть $\vee$. Так как $\mathscr{F} \neq \mathbf{1}$ (т.е. $\mathscr{P} \supset \mathscr{Q} \neq \mathbf{1}$ ), то, как и выше, получаем, что $\mathscr{F}=\mathscr{Q}$. Если $\mathscr{S} \supset \mathscr{T}=\mathbf{1}$, то $\mathscr{G}=\mathscr{S} \vee \mathscr{T}=\mathscr{T}$, и поэтому имеем $\mathscr{R}=(\mathscr{Q} \supset \mathscr{T}) \vee((\mathscr{Q} \supset \mathscr{T}) \supset \mathscr{Q})=\mathbf{1}$. Пусть $\mathscr{S} \supset \mathscr{T} \neq \mathbf{1}$. Тогда, как и в предыдушем случае, получаем, что $\mathscr{T} \supset \mathscr{S}=\mathbf{1}$. Значит, $\mathscr{G}=\mathscr{S} \vee \mathscr{T}=\mathscr{S}$. Следовательно,

$$
\mathscr{R}=(\mathscr{Q} \supset \mathscr{S}) \vee((\mathscr{Q} \supset \mathscr{S}) \supset \mathscr{Q})=\mathbf{1} .
$$

Случай, когда о есть $\&$, a $*$ есть $\supset$, рассматривается аналогично случаю, когда $\circ$ есть $\supset$, а $*$ есть \&.

Пусть ○ и $*$ суть \&. Если $\mathscr{P} \supset \mathscr{Q}=\mathbf{1}$ и $\mathscr{S} \supset \mathscr{T}=\mathbf{1}$, то $\mathscr{F}=$ $\mathscr{P} \& \mathscr{Q}=\mathscr{P}$ и $\mathscr{G}=\mathscr{S} \& \mathscr{T}=\mathscr{S}$, а поэтому имеем $\mathscr{R}=(\mathscr{P} \supset \mathscr{S}) \vee((\mathscr{P} \supset$ $\mathscr{S}) \supset \mathscr{P})=1$. Пусть $\mathscr{P} \supset \mathscr{Q}=\mathbf{1}$ и $\mathscr{S} \supset \mathscr{T} \neq \mathbf{1}$. Тогда, как и выше, получаем, что $\mathscr{T} \supset \mathscr{S}=1$. Значит, $\mathscr{F}=\mathscr{P} \& \mathscr{Q}=\mathscr{P}$ и $\mathscr{G}=\mathscr{S} \& \mathscr{T}=\mathscr{T}$. Следовательно, $\mathscr{R}=(\mathscr{P} \supset \mathscr{T}) \vee((\mathscr{P} \supset \mathscr{T}) \supset \mathscr{P})=\mathbf{1}$. Если $\mathscr{P} \supset \mathscr{Q} \neq \mathbf{1}$ и $\mathscr{S} \supset \mathscr{T}=\mathbf{1}$, то аналогичными рассуждениями получаем, что $\mathscr{R}=1$. Пусть $\mathscr{P} \supset \mathscr{Q} \neq 1$ и $\mathscr{S} \supset \mathscr{T} \neq \mathbf{1}$. Тогда, как и выше, получаем, что $\mathscr{Q} \supset \mathscr{P}=\mathbf{1}$ и $\mathscr{T} \supset \mathscr{S}=\mathbf{1}$. Значит, $\mathscr{F}=\mathscr{P} \& \mathscr{Q}=\mathscr{Q}$ и $\mathscr{G}=\mathscr{S} \& \mathscr{T}=\mathscr{T}$. Следовательно,

$$
\mathscr{R}=(\mathscr{Q} \supset \mathscr{T}) \vee((\mathscr{Q} \supset \mathscr{T}) \supset \mathscr{Q})=\mathbf{1} .
$$

Аналогичным образом рассматриваются следующие случаи: о есть \&, a * есть $\vee$; ○ есть $\vee$, a $*$ есть $\&$; о и $*$ суть $\vee$. Случай, когда о есть $\vee, a *$ есть $\supset$, рассматривается аналогично случаю, когда о есть $\supset$, a * есть $\vee$. Лемма доказана.

С помощью теоремы 1 и леммы 2 легко получаются результаты работы [1] о том, что исчисления $\mathbf{H}+\{\neg b \vee \neg \neg b),(c \supset d) \vee((c \supset d) \supset c)\}, \mathbf{H}+\{b \vee \neg b\}$ и $\mathbf{H}+\{\neg b \vee \neg \neg b\}$ обладают свойством простой подстановки. Поскольку они являются аксиоматизациями логик $\mathfrak{L}((b \supset d) \vee(d \supset b))$ (предтабличной), $\mathfrak{L}(b \vee \neg b)$ (классической) и $\mathfrak{L}(\neg b \vee \neg \neg b)$ соответственно, то эти логики обладают таким же свойством.

\section{§3. Логика $\mathfrak{L}((\neg b \supset(c \vee d)) \supset((\neg b \supset c) \vee(\neg b \supset d)))$}

В этом параграфе докажем анонсированную в [9] теорему 2 о том, что логика $\mathfrak{L}((\neg b \supset(c \vee d)) \supset((\neg b \supset c) \vee(\neg b \supset d)))$ (см. [11]) не обладает свойством простой подстановки. Наряду с [11] она изучалась и в других работах, например в [27]. В дальнейшем формулу $(\neg b \supset(c \vee d)) \supset((\neg b \supset c) \vee(\neg b \supset d))$ будем обозначать буквой $K$.

Пусть $n \geqslant 1$. Построим $\supset$-алгебру $\Xi_{n}$ следующим образом. Возьмем множество $\Delta_{n}=\left\{\alpha_{1}, \ldots, \alpha_{n}\right\}$. Каждому подмножеству $\left\{\alpha_{i_{1}}, \ldots, \alpha_{i_{m}}\right\}$, где $1 \leqslant i_{1}<\cdots$ $\cdots<i_{m} \leqslant n$, множества $\Delta_{n}$ поставим в соответствие объект $\alpha_{i_{1}} \vee \ldots \vee \alpha_{i_{m}}$. Положим $\Upsilon_{n}=\left\{\alpha_{i_{1}} \vee \ldots \vee \alpha_{i_{m}} \mid 1 \leqslant i_{1}<\cdots<i_{m} \leqslant n\right\}$, а $\Xi_{n}=\Upsilon_{n} \cup\{\beta, \gamma, \mathbf{1}\}$. На множестве $\Xi_{n}$ определим отношение частичного порядка $\leqslant$ так, как изображено на рис. 1 (на рис. 2 для $n=2$ ), причем меньшие элементы расположены ниже, чем бо́льшие.

Для любых $\xi$ и $\eta$ из $\Xi_{n}$ положим $\xi \supset \eta=\mathbf{1}$, если $\xi \leqslant \eta$, и $\xi \supset \eta=\eta$ в противном случае. По лемме 1 из [27] множество $\Xi_{n}$ вместе с этой операцией и выделенным элементом, равным 1, будет $\supset$-алгеброй. Прослеживая построение $\supset$-алгебры $\Xi_{n}$, можно заметить, что, по сути, на рис. 1 изображена ее схема. Поэтому в дальнейшем будем пользоваться этим фактом. 


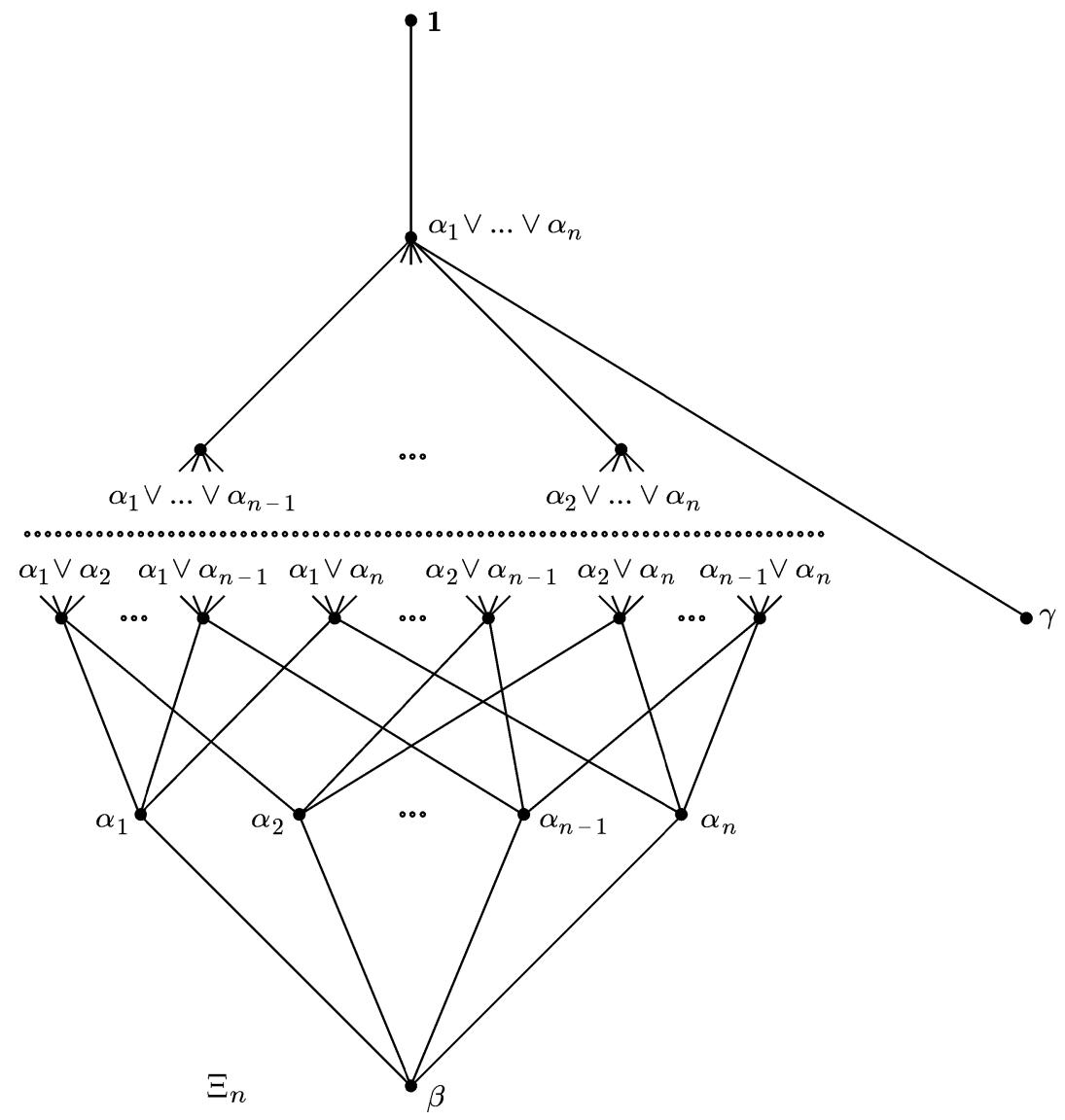

Рис. 1

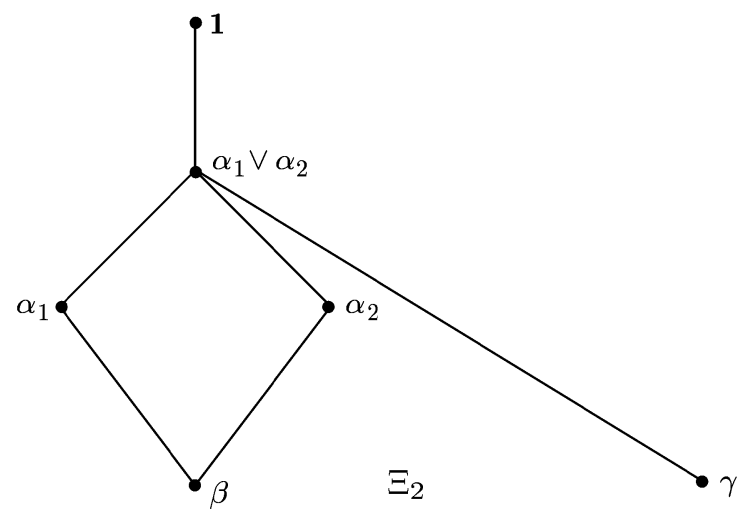

Рис. 2

Согласно [31, теорема 1] для $\Xi_{n}$ построим псевдобулеву алгебру $\Phi_{n}=I^{-1}\left(\Xi_{n}\right)$. Легко видеть, что алгебры $\Xi_{n}$ и $\Phi_{n}$ будут гёделевыми. Так как в силу [31, теорема 1] つ-алгебры $\Xi_{n}$ и $I\left(\Phi_{n}\right)$ изоморфны, то для простоты и удобства изложения материала отождествим соответствующие при выбранном изоморфизме элементы. Поэтому в дальнейшем будем считать, что $\supset$-алгебра $\Xi_{n}$ совпадает с 
$\supset$-подалгеброй $I\left(\Phi_{n}\right)$ псевдобулевой алгебры $\Phi_{n}$. Согласно построению $\supset$-алгебры $\Xi_{n}$ (см. рис. 1 ) и лемме 2 из [27] она является $\supset \vee$-алгеброй. Тогда по теореме 2 из [31] с учетом только что принятого соглашения получаем, что $\supset$-подалгебра $I\left(\Phi_{n}\right)$ (т. е. $\left.\Xi_{n}\right)$ алгебры $\Phi_{n}$ является ее $\supset \vee$-подалгеброй, совпадающей с $\supset \vee$-алгеброй $\Xi_{n}$. Этим фактом будем пользоваться в доказательствах лемм 3 и 4.

Элемент $\alpha_{1} \vee \ldots \vee \alpha_{n}$ алгебры $\Phi_{n}$ обозначим через $\omega_{n}$. Пусть $\Delta-$ подмножество множества $\Delta_{n}$. Положим $\Upsilon_{n, \Delta}=\varnothing$, если $\Delta=\varnothing$, и $\Upsilon_{n, \Delta}=\left\{\alpha_{i_{1}} \vee \ldots \vee \alpha_{i_{m}} \mid \alpha_{i_{1}} \in\right.$ $\left.\Delta, \ldots, \alpha_{i_{m}} \in \Delta, 1 \leqslant i_{1}<\cdots<i_{m} \leqslant n\right\}$, если $\Delta \neq \varnothing$, а $\Sigma_{n, \Delta}=\Upsilon_{n, \Delta} \cup$ $\left\{\beta, \gamma, \omega_{n}, \mathbf{1}\right\}$. Если $\Delta=\Delta_{n}$, то будет $\Upsilon_{n, \Delta}=\Upsilon_{n}$ и $\Sigma_{n, \Delta}=\Xi_{n}$.

ЛЕмма 3. Имеют место следующие утверждения:

1) әлементы подмножества $\{\beta\} \cup \Delta_{n}$ псевдобулевой алгебры $\Phi_{n}$ являются ее образуюшими ;

2) каждая псевдобулева подалгебра псевдобулевой алгебры $\Phi_{2}$, порожсднная одним әлементом, изоморфна алгебре, схема которой изображена на одном из рис. 3-6.

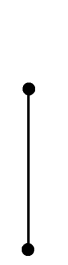

Рис. 3

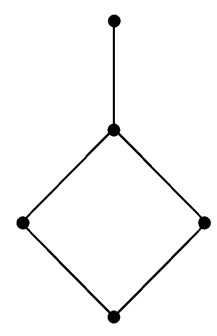

Рис. 5

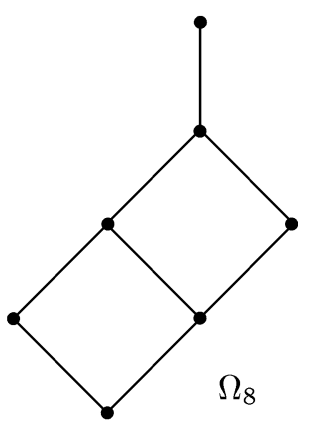

Рис. 6

ДокаЗАТЕЛьство. Докажем утверждение 1). Согласно построению алгебры $\Phi_{n}$ имеем $I\left(\Phi_{n}\right)=\Xi_{n}$. Поскольку каждый элемент алгебры $\Phi_{n}$ представляется в виде пересечения элементов из $\Xi_{n}$, то для нашей цели достаточно доказать, что в $\Phi_{n}$ элементы множества $\Xi_{n}$ выражаются через элементы множества $\{\beta\} \cup \Delta_{n}$. Легко видеть, что $\beta \supset \beta=\mathbf{1}$. Так как $\supset$-подалгебра $I\left(\Phi_{n}\right)$ алгебры $\Phi_{n}$ является ее $\supset \vee$-подалгеброй, совпадаюшей с $\supset \vee$-алгеброй $\Xi_{n}$, то в $\Phi_{n}$ каждый элемент из $I\left(\Phi_{n}\right) \backslash\{\mathbf{1}, \beta, \gamma\}$ получается из элементов множества $\Delta_{n}$ с помощью операции объединения. Согласно построению алгебры $\Phi_{n}$ и $[17$, лемма 5,1$\left.)\right]$ имеем $r(\mathbf{0})=\{\beta, \gamma\}$. Следовательно, $\neg \beta=\beta \supset \mathbf{0}=\gamma$.

Докажем утверждение 2$)$. Пусть $\xi \in \Phi_{2}$ и $\Theta$ - псевдобулева подалгебра алгебры $\Phi_{2}$, порожденная элементом $\xi$. Непосредственно из построения алгебры $\Phi_{2}$ вытекают следующие утверждения. Если $\xi \in\{\mathbf{1}, \mathbf{0}\}$, то $\Theta$ изоморфна псевдобулевой алгебре, изображенной на рис. 3. Если $\xi \in\left\{\alpha_{1} \vee \alpha_{2}, \alpha_{1}, \alpha_{2}, \alpha_{1} \& \alpha_{2}\right\}$, то $\Theta$ изоморфна псевдобулевой алгебре, изображенной на рис. 4. Если $\xi \in\{\beta, \gamma\}$, то $\Theta$ изоморфна псевдобулевой алгебре, изображенной на рис. 5. Если $\xi \in\left\{\alpha_{1} \& \gamma, \alpha_{2} \& \gamma\right.$, $\left.\alpha_{1} \& \alpha_{2} \& \gamma\right\}$, то $\Theta$ изоморфна псевдобулевой алгебре, изображенной на рис. 6 . Лемма доказана. 
Лемма 4. Пусть $X$ - пропозиииональная формула, построенная из переменных $b_{1}, \ldots, b_{n}, f$ - оценка переменных $b_{1}, \ldots, b_{n}$ в алгебре $\Phi_{n}$, a $\Delta$ - подмножество множества $\Delta_{n}$. Тогда верны следующие утвержсдения:

1) ecлu $f\left(b_{i}\right) \in\{\beta\} \cup \Delta, \quad 1 \leqslant i \leqslant n$, mor $r(f(X)) \subseteq \Sigma_{n, \Delta}$;

2) если $f\left(b_{i}\right) \in \Delta, 1 \leqslant i \leqslant n$, mо $f(X)=\mathbf{0}$ или $f(X) \geqslant \bar{\Delta}$.

ДоказАТЕльство. Пусть заданы $X, f$ и $\Delta$, удовлетворяющие условию леммы. Докажем утверждение 1$)$. Пусть $f\left(b_{i}\right) \in\{\beta\} \cup \Delta, 1 \leqslant i \leqslant n$. Индукцией по построению формулы $X$ покажем, что $r(f(X)) \subseteq \Sigma_{n, \Delta}$. Пусть $X$ есть $b_{j}$, где $1 \leqslant j \leqslant n$. Тогда $r\left(f\left(b_{j}\right)\right)=\left\{f\left(b_{j}\right)\right\}$. Поскольку $\{\beta\} \cup \Delta \subseteq \Sigma_{n, \Delta}$, имеем $r(f(X)) \subseteq \Sigma_{n, \Delta}$.

Пусть $X$ есть $Y \circ Z$, где $Y$ и $Z$ - пропозициональные формулы, а о - один из знаков $\supset$, или $\&$, или $\vee$. Пусть $r(f(Y)) \subseteq \Sigma_{n, \Delta}$ и $r(f(Z)) \subseteq \Sigma_{n, \Delta}$. Легко видеть, что $f(X)=f(Y) \circ f(Z)$. Если о есть つ, то, поскольку $r(f(Y) \supset f(Z))=\{\mathbf{1}\}$ или $r(f(Y) \supset f(Z)) \subseteq r(f(Z))$, имеем $r(f(X)) \subseteq \Sigma_{n, \Delta}$. Если о есть \&, то, поскольку $r(f(Y) \& f(Z)) \subseteq r(f(Y)) \cup r(f(Z))$, имеем $r(f(X)) \subseteq \Sigma_{n, \Delta}$.

Пусть о есть $\vee$. Нетрудно проверить, что

$$
f(Y) \vee f(Z)=\overline{\{\xi \vee \eta \mid \xi \in r(f(Y)), \eta \in r(f(Z))\}} .
$$

Пусть $\xi \in r(f(Y))$ и $\eta \in r(f(Z))$. Докажем, что $\xi \vee \eta \in \Sigma_{n, \Delta}$. Поскольку $\supset$-подалгебра $I\left(\Phi_{n}\right)$ алгебры $\Phi_{n}$ является ее $\supset \vee$-подалгеброй, совпадающей с $\supset \vee$-алгеброй $\Xi_{n}$, то в $\Phi_{n}$ выражение $\xi \vee \eta$ принимает следующее значение. Если $\xi=\mathbf{1}$ или $\eta=\mathbf{1}$, то имеем $\xi \vee \eta=\mathbf{1}$. Если $\xi=\omega_{n}$ и $\eta \in \Upsilon_{n, \Delta} \cup\left\{\beta, \gamma, \omega_{n}\right\}$, либо $\xi \in \Upsilon_{n, \Delta} \cup\{\beta, \gamma\}$ и $\eta=\omega_{n}$, то имеем $\xi \vee \eta=\omega_{n}$. Если $\xi=\gamma$ и $\eta \in \Upsilon_{n, \Delta} \cup\{\beta\}$, либо $\xi \in \Upsilon_{n, \Delta} \cup\{\beta\}$ и $\eta=\gamma$, то имеем $\xi \vee \eta=\omega_{n}$. Если $\xi=\eta=\gamma$, то имеем $\xi \vee \eta=\gamma$. Если $\xi=\beta$ и $\eta \in \Upsilon_{n, \Delta} \cup\{\beta\}$ или $\xi \in \Upsilon_{n, \Delta} \cup\{\beta\}$ и $\eta=\beta$, то имеем $\xi \vee \eta=\eta$ или $\xi \vee \eta=\xi$. Если $\xi, \eta \in \Upsilon_{n, \Delta}$, то $\xi=\alpha_{l_{1}} \vee \ldots \vee \alpha_{l_{m}}$ и $\eta=\alpha_{k_{1}} \vee \ldots \vee \alpha_{k_{s}}$, где $a_{l_{i}} \in \Delta$, $1 \leqslant i \leqslant m, \alpha_{k_{j}} \in \Delta, 1 \leqslant j \leqslant s, 1 \leqslant l_{1}<\cdots<l_{m} \leqslant n, 1 \leqslant k_{1}<\cdots<k_{s} \leqslant n$, a поэтому имеем $\xi \vee \eta=\alpha_{t_{1}} \vee \ldots \vee \alpha_{t_{q}}$, где $\left\{t_{1}, \ldots, t_{q}\right\}=\left\{l_{1}, \ldots, l_{m}\right\} \cup\left\{k_{1}, \ldots, k_{s}\right\}$ и $1 \leqslant t_{1}<\cdots<t_{q} \leqslant n$. Следовательно, $\xi \vee \eta \in \Sigma_{n, \Delta}$. Согласно построению алгебры $\Phi_{n}$ имеем $\Sigma_{n, \Delta} \subseteq I\left(\Phi_{n}\right)$. Значит,

$$
r(f(Y) \vee f(Z)) \subseteq\{\xi \vee \eta \mid \xi \in r(f(Y)), \eta \in r(f(Z))\}
$$

В результате получаем, что $r(f(X)) \subseteq \Sigma_{n, \Delta}$.

Пусть $X$ есть $\neg Y$, где $Y$ - пропозициональная формула. Пусть $r(f(Y)) \subseteq \Sigma_{n, \Delta}$. Согласно построению алгебры $\Phi_{n}$ и $[17$, лемма 5,1$\left.)\right]$ имеем $r(\mathbf{0})=\{\beta, \gamma\}$. Пусть $\beta, \gamma \in r(f(Y))$. Тогда $f(Y)=\mathbf{0}$ и поэтому $f(X)=\mathbf{1}$, а следовательно, $r(f(X)) \subseteq$ $\Sigma_{n, \Delta}$.

Пусть $\beta \in r(f(Y))$ и $\gamma \notin r(f(Y))$. Так как $I\left(\Phi_{n}\right)$ есть $\Xi_{n}$ и в $\Xi_{n}$ каждьй элемент множества $\Xi_{n} \backslash\{\gamma\}$ сравним с $\beta$ (см. рис. 1 ), то $f(Y)=\beta$. Поэтому имеем $f(X)=\neg f(Y)=\neg \beta=\gamma$. Следовательно, $r(f(X)) \subseteq \Sigma_{n, \Delta}$.

Пусть $\beta \notin r(f(Y))$ и $\gamma \in r(f(Y))$. Согласно построению алгебры $\Phi_{n}$ для любого $\zeta$ из $r(f(Y))$ в ней верно равенство $\zeta \supset \beta=\beta$. Поскольку $\gamma \supset \mathbf{0}=\beta$, имеем $f(X)=\neg f(Y)=r(f(Y)) \supset \mathbf{0}=\beta$. Следовательно, $r(f(X)) \subseteq \Sigma_{n, \Delta}$.

Пусть $\beta \notin r(f(Y))$ и $\gamma \notin r(f(Y))$. Тогда для любого $\zeta$ из $r(f(Y))$ в $\Phi_{n}$ верно равенство $\zeta \supset \mathbf{0}=\mathbf{0}$. Значит, $f(X)=\neg f(Y)=r(f(Y)) \supset \mathbf{0}=\mathbf{0}$. Поскольку $r(\mathbf{0})=\{\beta, \gamma\}$, имеем $r(f(X)) \subseteq \Sigma_{n, \Delta}$. 
Докажем утверждение 2$)$. Пусть $f\left(b_{i}\right) \in \Delta, 1 \leqslant i \leqslant n$. Тогда имеем $\Delta \neq \varnothing$. Индукцией по построению формулы $X$ покажем, что $f(X)=\mathbf{0}$ или $f(X) \geqslant \bar{\Delta}$. Если $X$ есть $b_{j}$, где $1 \leqslant j \leqslant n$, то $f(X) \geqslant \bar{\Delta}$.

Пусть $X$ есть $Y \circ Z$, где $Y$ и $Z$ - пропозициональные формулы, а о - один из знаков $\supset$, или $\&$, или $\vee$. Пусть $f(Y)=\mathbf{0}$ или $f(Y) \geqslant \bar{\Delta}$, и пусть $f(Z)=\mathbf{0}$ или $f(Z) \geqslant \bar{\Delta}$. Пусть о есть $\supset$. Если $f(Y)=\mathbf{0}$, то $f(X)=\mathbf{1}$, и поэтому имеем $f(X) \geqslant$ $\bar{\Delta}$. Если $f(Z) \geqslant \bar{\Delta}$, то имеем $f(X)=f(Y) \supset f(Z) \geqslant f(Z) \geqslant \bar{\Delta}$. Пусть $f(Y) \geqslant \bar{\Delta}$ и $f(Z)=\mathbf{0}$. Тогда имеем $f(X)=f(Y) \supset f(Z) \leqslant \bar{\Delta} \supset \mathbf{0}=\mathbf{0}$. Следовательно, $f(X)=\mathbf{0}$.

Пусть о есть \&. Если $f(Y)=\mathbf{0}$ или $f(Z)=\mathbf{0}$, то имеем $f(X)=f(Y) \& f(Z)=\mathbf{0 .}$ Если $f(Y) \geqslant \bar{\Delta}$ и $f(Z) \geqslant \bar{\Delta}$, то имеем $f(X)=f(Y) \& f(Z) \geqslant \bar{\Delta}$.

Пусть $\circ$ есть $\vee$. Если $f(Y)=\mathbf{0}$ и $f(Z)=\mathbf{0}$, то имеем $f(X)=f(Y) \vee f(Z)=\mathbf{0 .}$ Если $f(Y) \geqslant \bar{\Delta}$ или $f(Z) \geqslant \bar{\Delta}$, то имеем $f(X)=f(Y) \vee f(Z) \geqslant f(Y) \geqslant \bar{\Delta}$ или $f(X)=f(Y) \vee f(Z) \geqslant f(Z) \geqslant \bar{\Delta}$.

Пусть $X$ есть $\neg Y$, где $Y$ - пропозициональная формула. Пусть $f(Y)=\mathbf{0}$ или $f(Y) \geqslant \bar{\Delta}$. Если $f(Y)=\mathbf{0}$, то $f(X)=\neg f(Y)=\mathbf{1}$, и поэтому имеем $f(X) \geqslant$ $\bar{\Delta}$. Пусть $f(Y) \geqslant \bar{\Delta}$. Тогда имеем $f(X)=\neg f(Y)=f(Y) \supset \mathbf{0} \leqslant \bar{\Delta} \supset \mathbf{0}=\mathbf{0}$. Следовательно, $f(X)=\mathbf{0}$. Лемма доказана.

Пусть $n \geqslant 1$ и $b_{1}, \ldots, b_{n}$ - различные пропозициональные переменные. Положим $\mathfrak{K}_{n}=\{(\neg X \supset(Y \vee Z)) \supset((\neg X \supset Y) \vee(\neg X \supset Z)) \mid X, Y, Z$ - пропозициональные формулы, построенные из переменных $\left.b_{1}, \ldots, b_{n}\right\}$.

ЛЕмма 5. Каждая формула из $\mathfrak{K}_{n}$ общезначима во множестве $\{\beta\} \cup \Delta_{n}$ псевдобулевой алгебры $\Phi_{n}$.

ДокаЗАТЕЛЬСтво. Пусть $S \in \mathfrak{K}_{n}$. Тогда имеем

$$
S=(\neg X \supset(Y \vee Z)) \supset((\neg X \supset Y) \vee(\neg X \supset Z)),
$$

где $X, Y, Z$-пропозициональные формулы, построенные из переменных $b_{1}, \ldots, b_{n}$. Пусть $f$ - оценка переменных $b_{1}, \ldots, b_{n}$ элементами множества $\{\beta\} \cup \Delta$. Покажем, что в $\Phi_{n}$ верно равенство $f(S)=1$. В силу построения алгебры $\Phi_{n}$ и $[17$, лемма 5,1$)]$ имеем $r(\mathbf{0})=\{\beta, \gamma\}$.

Рассмотрим случай, когда $\beta, \gamma \in r(f(X))$. Тогда $f(X)=\mathbf{0}$, поэтому $\neg f(X)=\mathbf{1}$, а следовательно,

$$
\begin{aligned}
f(S) & =(\neg f(X) \supset(f(Y) \vee f(Z))) \supset((\neg f(X) \supset f(Y)) \vee(\neg f(X) \supset f(Z))) \\
& =(\mathbf{1} \supset(f(Y) \vee f(Z))) \supset((\mathbf{1} \supset f(Y)) \vee(\mathbf{1} \supset f(Z))) \\
& =(f(Y) \vee f(Z)) \supset(f(Y) \vee f(Z))=\mathbf{1} .
\end{aligned}
$$

Рассмотрим случай, когда $\beta \in r(f(X))$ и $\gamma \notin r(f(X))$. Так как $I\left(\Phi_{n}\right)$ есть $\Xi_{n}$ и в $\Xi_{n}$ каждый элемент множества $\Xi_{n} \backslash\{\gamma\}$ сравним с $\beta$ (см. рис. 1 ), то $f(X)=\beta$. Поскольку $r(\mathbf{0})=\{\beta, \gamma\}$, имеем $\neg f(X)=f(X) \supset \mathbf{0}=\beta \supset \gamma=\gamma$. Докажем, что $f\left(b_{i}\right) \in\{\beta\} \cup \Delta, 1 \leqslant i \leqslant n, \Delta \subseteq \Delta_{n}$ и $\Delta \neq \Delta_{n}$. Для этого достаточно показать, что существует $l$, удовлетворяющее условиям $1 \leqslant l \leqslant n$ и $f\left(b_{l}\right)=\beta$. Предположим, что $f\left(b_{i}\right) \in \Delta_{n}$ для всякого $i, 1 \leqslant i \leqslant n$. Тогда в силу леммы 4,2$)$ имеем 
$f(X)=\mathbf{0}$ или $f(X) \geqslant \bar{\Delta}_{n}$. Значит, $\gamma \in r(f(X))$ или $\beta \notin r(f(X))$. Противоречие. Следовательно, найдется такое $l$, что $1 \leqslant l \leqslant n$ и $f\left(b_{l}\right)=\beta$. Тем самым получаем, что $f\left(b_{i}\right) \in\{\beta\} \cup \Delta, 1 \leqslant i \leqslant n, \Delta \subseteq \Delta_{n}$ и $\Delta \neq \Delta_{n}$. Поэтому в силу леммы 4,1$)$ имеем $r(f(Y)) \subseteq \Sigma_{n, \Delta}$ и $r(f(Z)) \subseteq \Sigma_{n, \Delta}$.

Если $\mathbf{1} \in r(f(Y))$ или $\mathbf{1} \in r(f(Z))$, то $f(Y)=\mathbf{1}$ или $f(Z)=\mathbf{1}$, а следовательно, $f(S)=\mathbf{1}$. Пусть $\omega_{n} \in r(f(Y))$ или $\omega_{n} \in r(f(Z))$. Тогда $f(Y)=\omega_{n}$ или $f(Z)=\omega_{n}$, а поэтому имеем $\neg f(X) \supset f(Y)=\gamma \supset \omega_{n}=\mathbf{1}$ или $\neg f(X) \supset f(Z)=\gamma \supset \omega_{n}=\mathbf{1}$. Значит, $f(S)=\mathbf{1}$.

Пусть $r(f(Y)) \subseteq \Upsilon_{n, \Delta} \cup\{\beta, \gamma\}$ и $r(f(Z)) \subseteq \Upsilon_{n, \Delta} \cup\{\beta, \gamma\}$. Если $r(f(Y))=\{\gamma\}$ или $r(f(Z))=\{\gamma\}$, то $\neg f(X) \supset f(Y)=\gamma \supset \gamma=\mathbf{1}$ или $\neg f(X) \supset f(Y)=\gamma \supset \gamma=\mathbf{1}$, а поэтому имеем $f(S)=\mathbf{1}$.

Пусть $r(f(Y)) \neq\{\gamma\}$ и $r(f(Z)) \neq\{\gamma\}$. Положим $\Theta=r(f(Y)) \backslash\{\gamma\}$ и $\Upsilon=$ $r(f(Z)) \backslash\{\gamma\}$. Тогда имеем $\Theta \neq \varnothing, \Upsilon \neq \varnothing, \Theta \subseteq \Upsilon_{n, \Delta} \cup\{\beta\}$ и $\subseteq \subseteq \Upsilon_{n, \Delta} \cup\{\beta\}$. Так как $\Delta \subseteq \Delta_{n}$ и $\Delta \neq \Delta_{n}$, то для любых $\xi$ из $\Theta$ и $\eta$ из $\Upsilon$ в $\Phi_{n}$ верно соотношение $\xi \vee \eta \in \Upsilon_{n, \Delta} \cup\{\beta\}$. Следовательно, $\gamma \supset \xi \vee \eta=\xi \vee \eta, \gamma \supset \xi=\xi$ и $\gamma \supset \eta=\eta$ для указанных $\xi$ и $\eta$. Поскольку $\neg f(X)=\gamma$, имеем

$$
\begin{aligned}
f(S) & =(\gamma \supset(f(Y) \vee f(Z))) \supset((\gamma \supset f(Y)) \vee(\gamma \supset f(Z))) \\
& =(\gamma \supset \overline{(r(f(Y))} \vee \overline{r(f(Z))})) \supset((\gamma \supset \overline{r(f(Y))}) \vee(\gamma \supset \overline{r(f(Z))})) \\
& =\overline{\{\gamma \supset \xi \vee \eta \mid \xi \in \Theta, \eta \in \Upsilon\}} \supset(\overline{\{\gamma \supset \xi \mid \xi \in \Theta\}} \vee \overline{\{\gamma \supset \eta \mid \eta \in \Upsilon\}}) \\
& =\overline{\{\xi \vee \eta \mid \xi \in \Theta, \eta \in \Upsilon\}} \supset \overline{(\{\xi \mid \xi \in \Theta\}} \vee \overline{\{\eta \mid \eta \in \Upsilon\}}) \\
& =\overline{\{\xi \vee \eta \mid \xi \in \Theta, \eta \in \Upsilon\}} \supset \overline{\{\xi \vee \eta \mid \xi \in \Theta, \eta \in \Upsilon\}}=\mathbf{1} .
\end{aligned}
$$

Рассмотрим случай, когда $\beta \notin r(f(X))$ и $\gamma \in r(f(X))$. Согласно построению алгебры $\Phi_{n}$ для любого $\zeta$ из $r(f(X))$ в ней верно равенство $\zeta \supset \beta=\beta$. Поскольку $\gamma \supset \mathbf{0}=\beta$, имеем $\neg f(X)=r(f(X)) \supset \mathbf{0}=\beta$. Так как $I\left(\Phi_{n}\right)$ совпадает с $\Xi_{n}$, то для любого $\xi$ из $\Xi_{n} \backslash\{\gamma\}$ в $\Phi_{n}$ верно равенство $\beta \supset \xi=\mathbf{1}$. Если $\gamma \notin$ $r(f(Y))$ или $\gamma \notin r(f(Z))$, то $\neg f(X) \supset f(Y)=\beta \supset f(Y)=\mathbf{1}$ или $\neg f(X) \supset f(Z)=$ $\beta \supset f(Z)=\mathbf{1}$, а следовательно, $f(S)=\mathbf{1}$. Пусть $\gamma \in r(f(Y))$ и $\gamma \in r(f(Z))$. Тогда $\gamma \in r(f(Y) \vee f(Z))$. Поскольку $\neg f(X)=\beta$, имеем

$$
\begin{aligned}
\neg f(X) \supset(f(Y) \vee f(Z)) & =\beta \supset(f(Y) \vee f(Z))=\beta \supset \gamma=\gamma, \\
\neg f(X) \supset f(Y) & =\beta \supset f(Y)=\beta \supset \gamma=\gamma, \\
\neg f(X) \supset f(Z) & =\beta \supset f(Z)=\beta \supset \gamma=\gamma .
\end{aligned}
$$

Следовательно, $f(S)=\mathbf{1}$.

Рассмотрим случай, когда $\beta \notin r(f(X))$ и $\gamma \notin r(f(X))$. Тогда $r(f(X)) \subseteq \Xi_{n} \backslash$ $\{\beta, \gamma\}$. Так как $r(\mathbf{0})=\{\beta, \gamma\}$, то для любого $\xi$ из $r(f(X))$ в $\Phi_{n}$ верно равенство $\xi \supset \mathbf{0}=\mathbf{0}$. Поэтому имеем $\neg f(X)=r(f(X)) \supset \mathbf{0}=\mathbf{0}$. Значит, $\neg f(X) \supset f(Y)=\mathbf{1}$. Следовательно, $f(S)=\mathbf{1}$. Лемма доказана.

Докажем теорему, касающуюся аксиоматизаций логики $\mathfrak{L}(K)$.

Теорема 2. Имеют место следующие утверждения:

1) каково бы ни было конечное подмножество $\mathfrak{X}$ множсества $\mathfrak{K}_{1}$, исчисление $\mathbf{H}+\mathfrak{X}$ не является аксиоматизачией логики $\mathfrak{L}(K) ;$ 
2) если $n \geqslant 1, \mathfrak{Y}$ - конечное подмножество множества $\mathfrak{K}_{n}$ и исчисление $\mathbf{H}+\mathfrak{Y}$ является аксиоматизаиией логики $\mathfrak{L}(K)$, то $\mathbf{H}+\mathfrak{Y}$ не обладает свойством простой подстановки.

ДоказАтельство. Докажем утверждение 1). Пусть $\mathfrak{X}$ - конечное подмножество множества $\mathfrak{K}_{1}$. Для нашей цели достаточно показать, что формула $K$ не выводима в Н + $\mathfrak{X}$. По лемме 3 всякая псевдобулева подалгебра алгебры $\Phi_{2}$, порожденная одним элементом, изоморфна псевдобулевой алгебре, схема которой изображена на одном из рис. $3-6$. В силу [27, лемма 17$]$ формула $K$ обшезначима в каждой из этих алгебр. Тогда согласно построению множества $\mathfrak{K}_{1}$ всякая его формула общезначима в них. Так как множество $\mathfrak{K}_{1}$ содержит формулы только от одной переменной, то всякая его формула общезначима в $\Phi_{2}$. Поэтому все формулы множества $\mathfrak{X}$ общезначимы в $\Phi_{2}$. Построим оценку $f$ для переменных $b, c$ и $d$, положив $f(b)=\beta$, $f(c)=\alpha_{1}$ и $f(d)=\alpha_{2}$. Нетрудно проверить, что для оценки $f$ формула $K$ опровержима в $\Phi_{2}$. Следовательно, $K$ не выводима в $\mathbf{H}+\mathfrak{X}$.

Докажем утверждение 2). Пусть $n \geqslant 1, \mathfrak{Y}$ - конечное подмножество множества $\mathfrak{K}_{n}$ и исчисление $\mathbf{H}+\mathfrak{Y}$ является аксиоматизацией логики $\mathfrak{L}(K)$. Тогда $\mathfrak{Y} \neq \varnothing$, а в силу утверждения 1 ) этой теоремы имеем $n \geqslant 2$. Предположим, что исчисление $\mathbf{H}+\mathfrak{Y}$ обладает свойством простой подстановки. Тогда исчисление $\mathbf{H}+\{\overline{\mathfrak{Y}}\}$ является аксиоматизацией логики $\mathfrak{L}(K)$ и обладает этим свойством. Поэтому по теореме 1 формула $\overline{\mathfrak{Y}}$ наследственно обшезначима в $\Phi_{n}$. По лемме 5 каждая формула множества $\mathfrak{Y}$ общезначима во множестве $\{\beta\} \cup \Delta_{n}$ псевдобулевой алгебры $\Phi_{n}$. Значит, и формула $\bar{Y}$ обладает этим же свойством. По лемме 3 элементы множества $\{\beta\} \cup \Delta_{n}$ являются образуюшими алгебры $\Phi_{n}$. Тогда формула $\overline{\mathfrak{Y}}$ обшезначима в $\Phi_{n}$. Так как исчисление $\mathbf{H}+\{\overline{\mathfrak{Y}}\}$ - аксиоматизация логики $\mathfrak{L}(K)$, то формула $K$ выводима в нем и поэтому общезначима в $\Phi_{n}$. Построим оценку $g$ для переменных $b, c$ и $d$, положив $g(b)=\beta, g(c)=\alpha_{1}$ и $g(d)=\alpha_{2} \vee \ldots \vee \alpha_{n}$. Легко видеть, что

$$
\begin{aligned}
g(K) & =(\neg g(b) \supset(g(c) \vee g(d))) \supset((\neg g(b) \supset g(c)) \vee(\neg g(b) \supset g(d))) \\
& =\left(\neg \beta \supset\left(\alpha_{1} \vee\left(\alpha_{2} \vee \ldots \vee \alpha_{n}\right)\right)\right) \supset\left(\left(\neg \beta \supset \alpha_{1}\right) \vee\left(\neg \beta \supset\left(\alpha_{2} \vee \ldots \vee \alpha_{n}\right)\right)\right) \\
& =\left(\gamma \supset \omega_{n}\right) \supset\left(\left(\gamma \supset \alpha_{1}\right) \vee\left(\gamma \supset\left(\alpha_{2} \vee \ldots \vee \alpha_{n}\right)\right)\right) \\
& =\mathbf{1} \supset\left(\alpha_{1} \vee\left(\alpha_{2} \vee \ldots \vee \alpha_{n}\right)\right)=\omega_{n} .
\end{aligned}
$$

Тогда формула $K$ опровержима в $\Phi_{n}$. Противоречие. Следовательно, исчисление $\mathbf{H}+\mathfrak{Y}$ не обладает свойством простой подстановки. Теорема доказана.

ТЕорема 3 [9, теорема 2]. Логика $\mathfrak{L}(K)$ не обладает свойством простой подстановки.

ДокАЗАТЕльСТво. Предположим, что логика $\mathfrak{L}(K)$ обладает свойством простой подстановки. Тогда по лемме 1 существуют такие пропозициональные формулы $X_{1}, Y_{1}, Z_{1}, \ldots, X_{m}, Y_{m}, Z_{m}$, что исчисление

$$
\mathbf{H}+\left\{\mathbf{F}\left(b, c, d\left|X_{i}, Y_{i}, Z_{i}\right| K\right) \mid 1 \leqslant i \leqslant m\right\}
$$

является аксиоматизацией логики $\mathfrak{L}(K)$ и обладает этим свойством. Пусть формулы $X_{i}, Y_{i}, Z_{i}$, где $1 \leqslant i \leqslant m$, построены из переменных $d_{1}, \ldots, d_{n}$. Построим множество $\mathfrak{Y}$, заменив в формулах множества $\left\{\mathbf{F}\left(b, c, d\left|X_{i}, Y_{i}, Z_{i}\right| K\right) \mid 1 \leqslant i \leqslant m\right\}$ переменные $d_{1}, \ldots, d_{n}$ на переменные $b_{1}, \ldots, b_{n}$ соответственно. Легко видеть, что $\mathfrak{Y}-$ 
конечное подмножество множества $\mathfrak{K}_{n}$ и исчисление $\mathbf{H}+\mathfrak{Y}$ является аксиоматизацией логики $\mathfrak{L}(K)$ и обладает свойством простой подстановки, что противоречит теореме 2. Следовательно, логика $\mathfrak{L}(K)$ не обладает свойством простой подстановки. Теорема доказана.

\section{§ 4. Логики, включенные в логику $\mathfrak{L}(\neg \neg b \vee(\neg \neg b \supset b))$}

В этом параграфе докажем анонсированную в [9] теорему 3 о том, что если пропозищиональная формула $Y$ принадлежит логике $\mathfrak{L}(\neg \neg b \vee(\neg \neg b \supset b))$ и опровержима в определенной в [6] псевдобулевой алгебре (т. е. алгебре классов эквивалентности в Н формул от одной переменной), то логика $\mathfrak{L}(Y)$ не обладает свойством простой подстановки. Покажем [9, следствие 1], что логики, порожденные формулами от одной переменной и отличные от абсолютно противоречивой логики и логик $\mathfrak{L}(b \vee \neg b)$ и $\mathfrak{L}(\neg b \vee \neg \neg b)$, не обладают этим свойством.

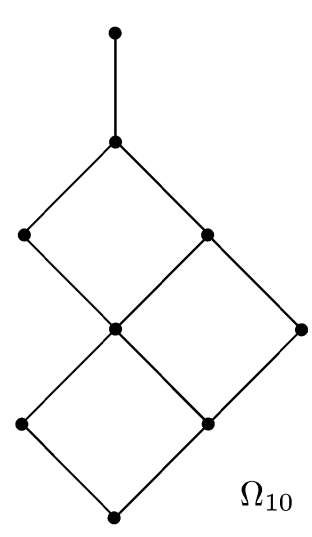

Рис. 7

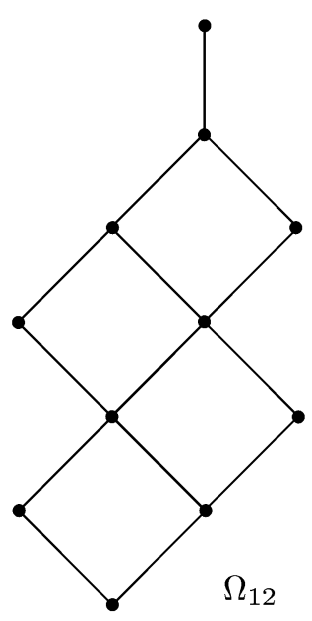

Рис. 8

Рассмотрим пропозициональные формулы $P_{0}, P_{1}, \ldots$, определенные в работе [6] следующими условиями: $P_{0}=b \& \neg b, P_{1}=b, P_{2}=\neg b, P_{2 i+3}=P_{2 i+1} \vee P_{2 i+2}$, $P_{2 i+4}=P_{2 i+3} \supset P_{2 i+1}$. Каждая пропозициональная формула от одной переменной $b$ эквивалентна в $\mathbf{H}$ некоторой из формул $b \supset b, P_{0}, P_{1}, \ldots[6]$. На множестве этих формул можно задать операции $\supset$ (относительного псевдодополнения), $\&$ (пересечения), $\vee$ (объединения) и $\neg$ (псевдодополнения) и, тем самым, превратить его в псевдобулеву алгебру [6], которую обозначим буквой $\Omega$. Отношение эквивалентности на алгебре $\Omega$, определяемое фильтром, порожденным элементом $P_{i}$, является конгруэнщией на $\Omega$ и поэтому задает псевдобулеву алгебру классов эквивалентности [23], которую обозначим символом $\Omega_{i}$.

Зафиксируем натуральное число $k$ такое, что $k \geqslant 4$. Рассмотрим гёделеву псевдобулеву алгебру $\Omega_{2 k}$ и ее $\supset$-подалгебру $I\left(\Omega_{2 k}\right)$. Для $k=4,5,6$ их схемы изображены на рис. 6-11. Легко видеть, что $I\left(\Omega_{2 k}\right)$ - частично упорядоченное множество с отношением порядка $\xi \leqslant \eta$, где $\xi \leqslant \eta \Longleftrightarrow \xi \supset \eta=1$. Множество $\mathfrak{M}\left(I\left(\Omega_{2 k}\right)\right)$ минимальных элементов $\supset$-алгебры $I\left(\Omega_{2 k}\right)$ состоит из двух элементов $\alpha$ и $\delta$, причем $\delta$ удовлетворяет неравенству $\delta \leqslant \zeta$ для всякого $\zeta$ из $I\left(\Omega_{2 k}\right) \backslash\{\alpha\}$ (см. рис. $\left.9-11\right)$. 


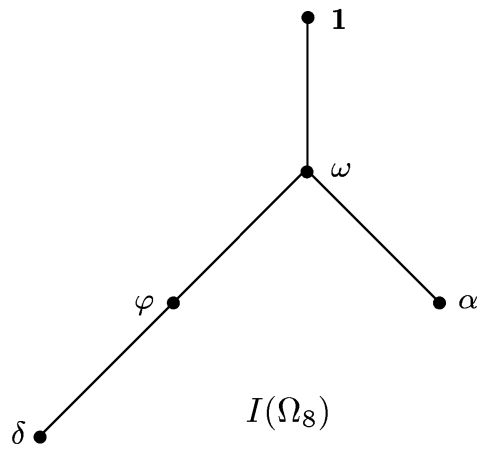

Рис. 9

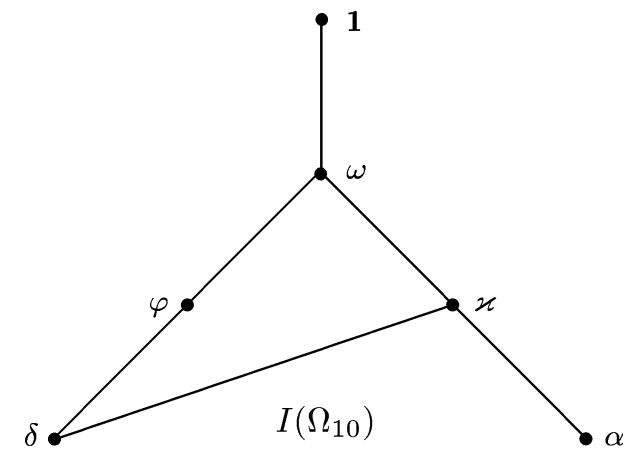

Рис. 10

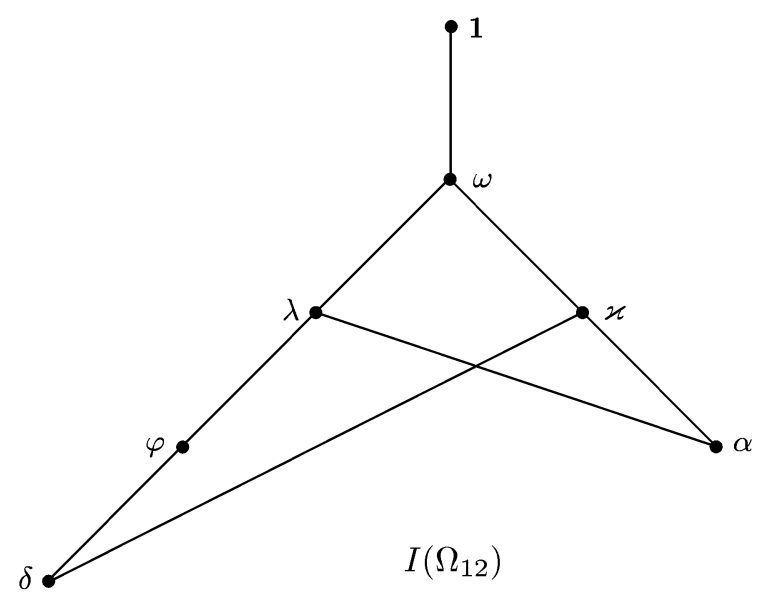

Рис. 11

В $\supset$-алгебре $I\left(\Omega_{2 k}\right)$ элемент $\delta$ является непосредственным предшественником одного элемента $\varphi$, если $k=4$, и двух элементов $\varphi$ и $\varkappa$, если $k \geqslant 5$.

Пусть $m \geqslant 1$. Для $\supset$-алгебры $I\left(\Omega_{2 k}\right)$ построим $\supset$-алгебру $\Psi_{k}^{m}$ следующим образом. Возьмем различные объекты $\delta_{1}, \ldots, \delta_{m}$, отличные от элементов множества $I\left(\Omega_{2 k}\right)$. Положим $\Psi_{k}^{m}=\left(I\left(\Omega_{2 k}\right) \backslash\{\delta\}\right) \cup\left\{\delta_{1}, \ldots, \delta_{m}\right\}$. С помощью определенного выше на $I\left(\Omega_{2 k}\right)$ отношения частичного порядка зададим на $\Psi_{k}^{m}$ бинарное отношение $\leqslant$. Пусть $\sigma, \tau \in I\left(\Omega_{2 k}\right) \backslash\{\delta\}$. Отношение $\sigma \leqslant \tau$ верно на $\Psi_{k}^{m}$, если $\sigma \leqslant \tau$ на $I\left(\Omega_{2 k}\right)$. Отношение $\delta_{i} \leqslant \tau$, где $1 \leqslant i \leqslant m$, верно на $\Psi_{k}^{m}$, если $\delta \leqslant \tau$ на $I\left(\Omega_{2 k}\right)$. На $\Psi_{k}^{m}$ верно отношение $\delta_{i} \leqslant \delta_{i}$, где $1 \leqslant i \leqslant m$. Нетрудно проверить, что отношение $\leqslant$ является отношением частичного порядка на $\Psi_{k}^{m}$, а выделенный элемент 1 алгебры $I\left(\Omega_{2 k}\right)$ - наибольшим элементом множества $\Psi_{k}^{m}$.

Для любых $\xi$ и $\eta$ из $\Psi_{k}^{m}$ положим $\xi \supset \eta=\mathbf{1}$, если $\xi \leqslant \eta$, и $\xi \supset \eta=\eta$ в противном случае. В силу $[27$, лемма 1$]$ множество $\Psi_{k}^{m}$ вместе с этой операцией и выделенным элементом, равным $\mathbf{1}$, будет $\supset$-алгеброй. Для $k=4,5,6$ их схемы изображены на рис. $12-14$.

Согласно [31, теорема 1] для $\supset$-алгебры $\Psi_{k}^{m}$ построим псевдобулеву алгебру $\Omega_{k}^{m}=I^{-1}\left(\Psi_{k}^{m}\right)$. Так как в силу этой теоремы $\supset$-алгебры $\Psi_{k}^{m}$ и $I\left(\Omega_{k}^{m}\right)$ изоморф- 


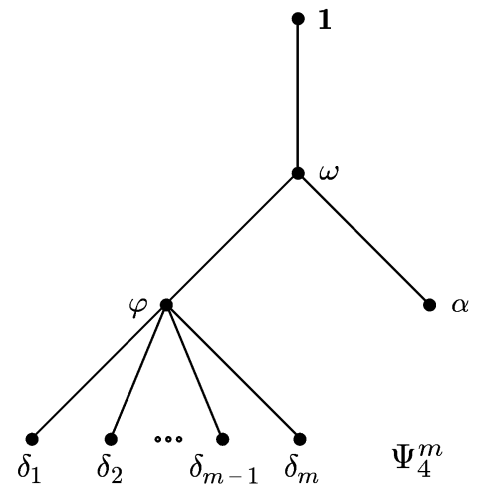

Рис. 12

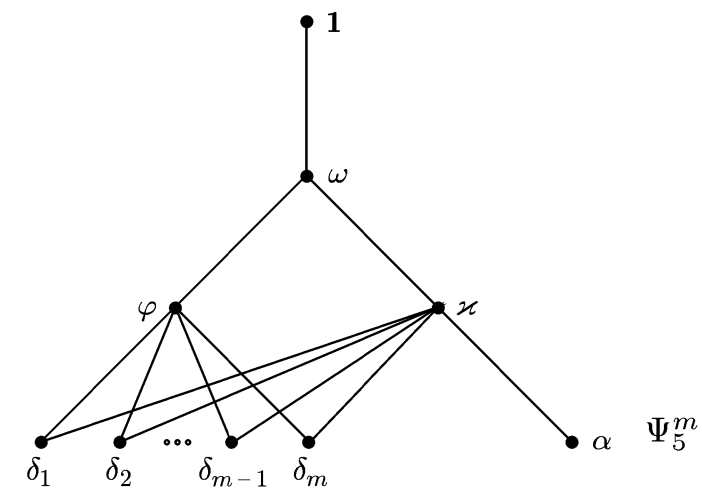

Рис. 13

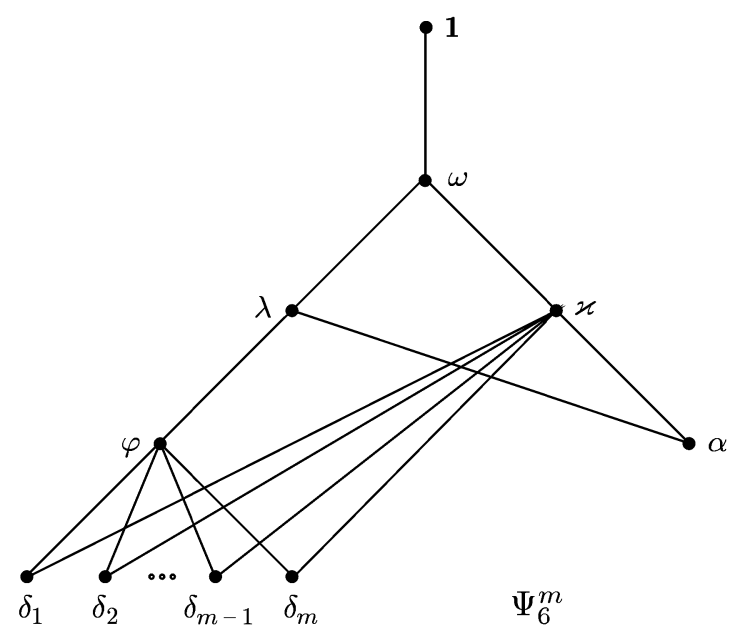

Рис. 14

ны, то для простоты и удобства изложения материала отождествим соответствуюшие при выбранном изоморфизме элементы. Поэтому в дальнейшем будем считать, что $\supset$-алгебра $\Psi_{k}^{m}$ совпадает с $\supset$-подалгеброй $I\left(\Omega_{k}^{m}\right)$ псевдобулевой алгебры $\Omega_{k}^{m}$. Легко видеть, что как $\supset$-алгебры $\Psi_{k}^{1}$ и $I\left(\Omega_{2 k}\right)$, так и псевдобулевы алгебры $\Omega_{k}^{1}$ и $\Omega_{2 k}$ изоморфны.

Пусть $n \geqslant 1$. Число слов в алфавите $\{0,1\}$ длины $n+1$, отличных от слова, состоящего из одних нулей, и слова, состоящего из одних единиц, равно $l=2^{n+1}-2$. Занумеруем их в каком-нибудь порядке. Пусть это будут $E_{1}, \ldots, E_{l}$. Для $i$ и $j$, удовлетворяющих условиям $1 \leqslant i \leqslant n+1$ и $1 \leqslant j \leqslant l$, определим элементы $\varepsilon_{i}^{j}$ и $\varepsilon_{i}$ псевдобулевой алгебры $\Omega_{k}^{l}$, где $k \geqslant 4$, положив $\varepsilon_{i}^{j}=\mathbf{1}$, если $i$-я буква слова $E_{j}$ есть 1, и $\varepsilon_{i}^{j}=\delta_{j}$, если она есть 0, и положив $\varepsilon_{i}=\varepsilon_{i}^{1} \& \ldots \& \varepsilon_{i}^{l}$.

Докажем следующую лемму.

Лемма 6. Пусть $k \geqslant 4$. Тогда имеют место следующие утвержсдения: 1) псевдобулева алгебра $\Omega_{2 k}$ вложима в псевдобулеву алгебру $\Omega_{k}^{s}$ при любом натуральном числе $s$, удовлетворяющем условию $s \geqslant 1$; 
2) если $m \geqslant 2$, то элементы $\delta_{1}, \ldots, \delta_{m}$ псевдобулевой алгебры $\Omega_{k}^{m}$ являются ее образующими;

3) если $n \geqslant 1$ и $l=2^{n+1}-2$, то әлементы $\varepsilon_{1}, \ldots, \varepsilon_{n+1}$ псевдобулевой алгебры $\Omega_{k}^{l}$ являются ее образующими.

ДокаЗАтельство. Пусть $k \geqslant 4$. Докажем утверждение 1$)$. Пусть $s \geqslant 1$. Построим отображение $f$ из $\supset$-алгебры $I\left(\Omega_{2 k}\right)$ в псевдобулеву алгебру $\Omega_{k}^{s}$, положив $f(\zeta)=\zeta$, если $\zeta \neq \delta$, и $f(\zeta)=\delta_{1} \& \ldots \& \delta_{s}$, если $\zeta=\delta$. Так как в $I\left(\Omega_{2 k}\right)$ верно неравенство $\delta \neq \mathbf{1}$, то $f(\mathbf{1})=\mathbf{1}$. Пусть $\xi, \eta \in I\left(\Omega_{2 k}\right)$. Покажем, что $f$ сохраняет операцию $\supset$, т. е. $f(\xi \supset \eta)=f(\xi) \supset f(\eta)$.

Пусть в $I\left(\Omega_{2 k}\right)$ верно равенство $\xi \supset \eta=1$. Рассмотрим случай, когда $\xi \neq \delta$. Тогда $\eta \neq \delta$. Значит, $f(\xi)=\xi$ и $f(\eta)=\eta$. Так как $\xi \neq \delta$ и $\eta \neq \delta$, то согласно построению алгебры $\Omega_{k}^{s}$ в ней верно равенство $\xi \supset \eta=1$. Следовательно, в $\Omega_{k}^{s}$ имеет место соотношение $f(\xi \supset \eta)=\mathbf{1}=f(\xi) \supset f(\eta)$.

Рассмотрим случай, когда $\xi=\delta$. Если $\eta=\delta$, то имеем $f(\xi \supset \eta)=f(\xi) \supset f(\eta)$. Пусть $\eta \neq \delta$. Тогда $f(\xi)=\delta_{1} \& \ldots \& \delta_{s}$ и $f(\eta)=\eta$. Так как в $I\left(\Omega_{2 k}\right)$ верно равенство $\xi \supset \eta=\mathbf{1}$, то в $\Omega_{k}^{s}$ верно равенство в $\delta_{i} \supset \eta=\mathbf{1}$, где $1 \leqslant i \leqslant s$. Следовательно, в $\Omega_{k}^{s}$ имеет место соотношение $f(\xi \supset \eta)=\mathbf{1}=\left(\delta_{1} \& \ldots \& \delta_{s}\right) \supset \eta=f(\xi) \supset f(\eta)$.

Пусть в $I\left(\Omega_{2 k}\right)$ верно неравенство $\xi \supset \eta \neq \mathbf{1}$. Тогда $\xi \supset \eta=\eta, \xi \neq \eta$ и $\eta \neq \mathbf{1}$. Рассмотрим случай, когда $\xi \neq \delta$ и $\eta \neq \delta$. Тогда имеем $f(\xi)=\xi$ и $f(\eta)=\eta$. Кроме того, в $\Omega_{k}^{s}$ верно равенство $\xi \supset \eta=\eta$. Следовательно, в $\Omega_{k}^{s}$ имеет место соотношение $f(\xi \supset \eta)=\eta=f(\xi) \supset f(\eta)$.

Рассмотрим случай, когда $\xi=\delta$ и $\eta \neq \delta$. Тогда имеем $f(\xi)=\delta_{1} \& \ldots \& \delta_{s}$ и $f(\eta)=\eta$. Так как в $\left.I\left(\Omega_{2 k}\right)\right)$ верно равенство $\xi \supset \eta=\eta$, то в $\Omega_{k}^{s}$ будет $\delta_{i} \supset \eta=\eta$, где $1 \leqslant i \leqslant s$. Следовательно, в $\Omega_{k}^{s}$ верно соотношение

$$
f(\xi \supset \eta)=\eta=\delta_{1} \supset\left(\ldots \supset\left(\delta_{s} \supset \eta\right) \cdots\right)=\left(\delta_{1} \& \ldots \& \delta_{s}\right) \supset \eta=f(\xi) \supset f(\eta) .
$$

Рассмотрим случай, когда $\xi \neq \delta$ и $\eta=\delta$. Тогда имеем $f(\xi)=\xi$ и $f(\eta)=$ $\delta_{1} \& \ldots \& \delta_{s}$. Согласно построению алгебры $\Omega_{k}^{s}$ в ней верно равенство $\xi \supset \delta_{i}=\delta_{i}$, где $1 \leqslant i \leqslant s$. Следовательно, в $\Omega_{k}^{s}$ имеет место соотношение

$$
\begin{aligned}
f(\xi \supset \eta) & =f(\eta)=\delta_{1} \& \ldots \& \delta_{s}=\left(\xi \supset \delta_{1}\right) \& \ldots \&\left(\xi \supset \delta_{s}\right) \\
& =\xi \supset\left(\delta_{1} \& \ldots \& \delta_{s}\right)=f(\xi) \supset f(\eta) .
\end{aligned}
$$

Таким образом, $f$ сохраняет операцию $\supset$. Легко видеть, что $f$ взаимно однозначно отображает $\supset$-алгебру $I\left(\Omega_{2 k}\right)$ на некоторую $\supset$-подалгебру псевдобулевой алгебры $\Omega_{k}^{s}$. Значит, $f$ - вложение $\supset$-алгебры $I\left(\Omega_{2 k}\right)$ в $\Omega_{k}^{s}$. Поэтому в силу $[32$, теорема 2,4$)]$ для доказательства утверждения 1) леммы 6 достаточно показать, что в $\Omega_{k}^{s}$ имеют место равенство $\overline{f\left(\mathfrak{M}\left(I\left(\Omega_{2 k}\right)\right)\right)}=\mathbf{0}$ и равенство $f^{\&}(\xi \vee \eta)=f^{\&}(\xi) \vee f^{\&}(\eta)$ для любых $\xi$ и $\eta$ из $I\left(\Omega_{2 k}\right)$.

Согласно построениям $\supset$-алгебр $I\left(\Omega_{2 k}\right)$ и $\Psi_{k}^{s}$ имеем $\mathfrak{M}\left(I\left(\Omega_{2 k}\right)\right)=\{\alpha, \delta\}$ и $\mathfrak{M}\left(\Psi_{k}^{s}\right)=\left\{\alpha, \delta_{1}, \ldots, \delta_{s}\right\}$ (см. рис. 9-14). Так как $I\left(\Omega_{k}^{s}\right)$ и $\Psi_{k}^{s}$ совпадают, то в силу $[17$, лемма 5$]$ получаем, что

$$
\overline{f\left(\mathfrak{M}\left(I\left(\Omega_{2 k}\right)\right)\right)}=\overline{f(\{\alpha, \delta\})}=\alpha \& \delta_{1} \& \ldots \& \delta_{s}=\mathbf{0} .
$$

Пусть $\xi, \eta \in I\left(\Omega_{2 k}\right)$. Если $\xi=\eta=\delta$, то имеем

$$
f^{\&}(\xi \vee \eta)=f^{\&}(\delta)=f(\delta)=\delta_{1} \& \ldots \& \delta_{s}=f(\xi) \vee f(\eta)=f^{\&}(\xi) \vee f^{\&}(\eta)
$$


Пусть $\xi \neq \delta$ или $\eta \neq \delta$. Тогда согласно построению алгебры $\Omega_{2 k}$ (см. рис. $6-11$ ) $\delta \notin r(\xi \vee \eta)$, и поэтому имеем $f(r(\xi \vee \eta))=r(\xi \vee \eta)$. В силу [32, лемма 1,1$)]$ в $\Omega_{2 k}$ имеет место равенство $r(\xi \vee \eta)=\mathfrak{M}\left(\mathfrak{k}\left(I\left(\Omega_{2 k}\right), \xi, \eta\right)\right)$. Покажем, что в $\Omega_{k}^{s}$ верно соотношение $r(f(\xi) \vee f(\eta))=\mathfrak{M}\left(\mathfrak{k}\left(I\left(\Omega_{2 k}\right), \xi, \eta\right)\right)$.

Рассмотрим случай, когда $\xi \neq \delta$ и $\eta \neq \delta$. Тогда $f(\xi)=\xi$ и $f(\eta)=\eta$. Поэтому в $\Omega_{k}^{s}$ верны равенства $r(f(\xi))=\{\xi\}$ и $r(f(\eta))=\{\eta\}$. А тогда в силу [32, лемма 1,3$)]$ в $\Omega_{k}^{s}$ верно соотношение $r(f(\xi) \vee f(\eta))=\mathfrak{M}\left(\mathfrak{k}\left(I\left(\Omega_{k}^{s}\right), \xi, \eta\right)\right)$. Так как $\xi \neq \delta$ и $\eta \neq \delta$, то согласно построению алгебры $\Omega_{k}^{s}$ имеем $\mathfrak{k}\left(I\left(\Omega_{2 k}\right), \xi, \eta\right)=\mathfrak{k}\left(I\left(\Omega_{k}^{s}\right), \xi, \eta\right)$ (см. рис. 9-14). Следовательно, $r(f(\xi) \vee f(\eta))=\mathfrak{M}\left(\mathfrak{k}\left(I\left(\Omega_{2 k}\right), \xi, \eta\right)\right)$.

Рассмотрим случай, когда $\xi \neq \delta$ и $\eta=\delta$. Тогда $f(\xi)=\xi$ и $f(\eta)=\delta_{1} \& \ldots \& \delta_{s}$. Поэтому в $\Omega_{k}^{s}$ верны равенства $r(f(\xi))=\{\xi\}$ и $r(f(\eta))=\left\{\delta_{1}, \ldots, \delta_{s}\right\}$, а тогда в силу $[32$, лемма 1,3$)]$ в $\Omega_{k}^{s}$ верно соотношение

$$
r(f(\xi) \vee f(\eta))=\mathfrak{M}\left(\bigcup_{i=1}^{s} \mathfrak{k}\left(I\left(\Omega_{k}^{s}\right), \xi, \delta_{i}\right)\right) .
$$

Так как $\xi \neq \delta$ и $\eta=\delta$, то согласно построению алгебры $\Omega_{k}^{s}$ имеем $\mathfrak{k}\left(I\left(\Omega_{2 k}\right), \xi, \eta\right)=$ $\mathfrak{k}\left(I\left(\Omega_{k}^{s}\right), \xi, \delta_{i}\right)$, где $1 \leqslant i \leqslant s$ (см. рис. 9-14). Следовательно,

$$
r(f(\xi) \vee f(\eta))=\mathfrak{M}\left(\mathfrak{k}\left(I\left(\Omega_{2 k}\right), \xi, \eta\right)\right) .
$$

Аналогичным образом рассматривается случай, когда $\xi=\delta$ и $\eta \neq \delta$. Таким образом, имеем $r(f(\xi) \vee f(\eta))=\mathfrak{M}\left(\mathfrak{k}\left(I\left(\Omega_{2 k}\right), \xi, \eta\right)\right)$. В результате получаем

$$
\begin{aligned}
f^{\&}(\xi \vee \eta) & =\overline{f(r(\xi \vee \eta))}=\overline{r(\xi \vee \eta)}=\overline{\mathfrak{M}\left(\mathfrak{k}\left(I\left(\Omega_{2 k}\right), \xi, \eta\right)\right)} \\
& =\overline{r(f(\xi) \vee f(\eta))}=f(\xi) \vee f(\eta)=f^{\&}(\xi) \vee f^{\&}(\eta) .
\end{aligned}
$$

Докажем утверждение 2). Пусть $m \geqslant 2$. Так как каждый элемент псевдобулевой алгебры $\Omega_{k}^{m}$ представляется в виде пересечения элементов из $I\left(\Omega_{k}^{m}\right)$ (т.е. из $\Psi_{k}^{m}$ ), то для нашей цели достаточно показать, что в $\Omega_{k}^{m}$ элементы множества $I\left(\Omega_{k}^{m}\right)$ выражаются через $\delta_{1}, \ldots, \delta_{m}$. Непосредственно из построения алгебры $\Omega_{k}^{m}$ вытекают следуюшие утверждения.

В $\Omega_{k}^{m}$ верны равенства $\delta_{1} \supset \delta_{1}=\mathbf{1}$ и $\neg\left(\delta_{1} \& \ldots \& \delta_{m}\right)=\alpha$ (см. рис. $\left.12-14\right)$.

Рассмотрим случай $k=4$ (см. рис. 12). В $\Omega_{4}^{m}$ верно равенство $\delta_{1} \vee \alpha=\omega$. Так как $m \geqslant 2$, то в $\Omega_{4}^{m}$ имеет место равенство $\delta_{1} \vee \delta_{2}=\varphi$.

Рассмотрим случай $k=5$ (см. рис. 13 ). Так как $m \geqslant 2$, то в $\Omega_{5}^{m}$ имеет место равенство $\delta_{1} \vee \delta_{2}=\varphi \& \varkappa$. Кроме того, в $\Omega_{5}^{m}$ верны соотношения

$$
\begin{gathered}
\alpha \supset\left(\delta_{1} \vee \delta_{2}\right)=\alpha \supset(\varphi \& \varkappa)=(\alpha \supset \varphi) \&(\alpha \supset \varkappa)=\varphi \& \mathbf{1}=\varphi \\
\varphi \supset\left(\delta_{1} \vee \delta_{2}\right)=(\varphi \supset \varphi) \&(\varphi \supset \varkappa)=\varkappa, \quad \varphi \vee \varkappa=\omega .
\end{gathered}
$$

Рассмотрим случай $k \geqslant 6$ (см. рис. 14 ). Поскольку $m \geqslant 2$, то в $\Omega_{k}^{m}$ имеет место равенство $\delta_{1} \vee \delta_{2}=\varphi \& \varkappa$. Кроме того, в $\Omega_{k}^{m}$ верны соотношения $\delta_{1} \vee \alpha=\lambda \& \varkappa$, $\alpha \supset\left(\delta_{1} \vee \delta_{2}\right)=\varphi, \varphi \supset\left(\delta_{1} \vee \delta_{2}\right)=\varkappa$ и $\supset\left(\delta_{1} \vee \alpha\right)=\varkappa \supset(\lambda \& \varkappa)=(\varkappa \supset \lambda) \&$ $(\varkappa \supset \varkappa)=\lambda$. Если

$$
\xi \in I\left(\Omega_{k}^{m}\right) \backslash\left(\left\{\delta_{1}, \ldots, \delta_{m}\right\} \cup\{\alpha, \varphi, \varkappa, \lambda\}\right)
$$


то в $\Omega_{k}^{m}$ элемент $\xi$ получаем из элементов $\varphi, \varkappa$ и $\lambda$ с помощью операций $\vee$ и $\supset$.

Докажем утверждение 3$)$. Пусть $n \geqslant 1$ и $l=2^{n+1}-2$. Тогда $l \geqslant 2$ и поэтому в силу утверждения 2) леммы для нашей цели достаточно показать, что в $\Omega_{k}^{l}$ элементы $\delta_{1}, \ldots, \delta_{l}$ выражаются через $\varepsilon_{1}, \ldots, \varepsilon_{n+1}$. Нетрудно проверить, что для всякого $i$, удовлетворяюшего условию $1 \leqslant i \leqslant n+1$, верны следующие утверждения:

i) среди слов $E_{1}, \ldots, E_{l}$ ровно у $l / 2$ слов $i$-я буква есть 0 ;

ii) $r\left(\varepsilon_{i}\right) \subseteq\left\{\delta_{1}, \ldots, \delta_{l}\right\}$;

iii) $\left|r\left(\varepsilon_{i}\right)\right|=l / 2$;

iv) если $1 \leqslant j \leqslant l$, то $\delta_{j} \in r\left(\varepsilon_{i}\right)$ тогда и только тогда, когда $i$-я буква слова $E_{j}$ есть 0 .

Пусть $1 \leqslant q \leqslant n+1$. Тогда найдется такое слово $E_{t}$, где $1 \leqslant t \leqslant l$, что его $q$-я буква есть 0 , а все остальные буквы суть 1 . Отсюда с помощью утверждения iv) получаем, что $\delta_{t} \in r\left(\varepsilon_{q}\right)$ и $\delta_{t} \notin r\left(\varepsilon_{j}\right)$, где $1 \leqslant j \leqslant n+1$ и $j \neq q$. Пусть $\delta_{u} \in r\left(\varepsilon_{q}\right)$ и $\delta_{u} \neq \delta_{t}$, где $1 \leqslant u \leqslant l$. Тогда в силу утверждения iv) $q$-я буква слова $E_{u}$ и еше не меньше одной его буквы равны 0. Поэтому имеем $\delta_{u} \in \bigcup_{j \in L} r\left(\varepsilon_{j}\right)$, где $L=\{1, \ldots, n+1\} \backslash\{q\}$. Следовательно, $\left(\left\{\varepsilon_{1}, \ldots, \varepsilon_{n+1}\right\} \backslash\left\{\varepsilon_{q}\right\}\right) \supset \varepsilon_{q}=\delta_{t}$.

Так как среди $E_{1}, \ldots, E_{l}$ имеются ровно $n+1$ слов, в которые буква 0 входит только один раз, то $n+1$ элемент множества $\left\{\delta_{1}, \ldots, \delta_{l}\right\}$ выразим через $\varepsilon_{1}, \ldots, \varepsilon_{n+1}$. Множество этих элементов обозначим через $\Theta$. Нетрудно проверить, что каждый элемент из $\Theta$ принадлежит некоторому из множеств $r\left(\varepsilon_{1}\right), \ldots, r\left(\varepsilon_{n+1}\right)$, причем различные элементы принадлежат различным множествам. Если $n=1$, то $\Theta=\left\{\delta_{1}, \ldots, \delta_{l}\right\}$ и утверждение 3 ) доказано. Пусть $n>1$. Тогда $\Theta \neq$ $\left\{\delta_{1}, \ldots, \delta_{l}\right\}$, и поэтому нам осталось выразить элементы множества $\left\{\delta_{1}, \ldots, \delta_{l}\right\} \backslash \Theta$ через $\varepsilon_{1}, \ldots, \varepsilon_{n+1}$. С этой целью положим $\iota_{i}=\Theta \supset \varepsilon_{i}$, где $1 \leqslant i \leqslant n+1$. Нетрудно проверить, что $l / 2 \geqslant 3,\left|r\left(\iota_{i}\right)\right|=(l / 2)-1$ и $r\left(\iota_{i}\right) \subseteq\left\{\delta_{1}, \ldots, \delta_{l}\right\} \backslash \Theta$.

Пусть $1 \leqslant p<s \leqslant n+1$. Так как $n>1$, то найдется такое слово $E_{v}$, где $1 \leqslant v \leqslant l$, что его $p$-я и $s$-я буквы суть 0 , а все остальные буквы суть 1 . Отсюда с помощью утверждения iv) получаем, что $\delta_{v} \in r\left(\varepsilon_{p}\right), \delta_{v} \in r\left(\varepsilon_{s}\right)$ и $\delta_{v} \notin r\left(\varepsilon_{j}\right)$, где $1 \leqslant j \leqslant n+1, j \neq p$ и $j \neq s$. Так как буква 0 входит в слово $E_{v}$ два раза, а в слова, соответствующие элементам из $\Theta$, один раз, то $\delta_{v} \notin \Theta$. Следовательно, $\delta_{v} \in r\left(\iota_{p}\right)$, $\delta_{v} \in r\left(\iota_{s}\right)$ и $\delta_{v} \notin r\left(\iota_{j}\right)$, где $1 \leqslant j \leqslant n+1, j \neq p$ и $j \neq s$.

Пусть $\delta_{z} \in r\left(\iota_{p}\right)$ и $\delta_{z} \neq \delta_{v}$, где $1 \leqslant z \leqslant l$. Тогда имеем $\delta_{z} \in r\left(\varepsilon_{p}\right)$ и $\delta_{z} \notin \Theta$. Поэтому $p$-я и $w$-я, где $1 \leqslant w \leqslant n+1, w \neq p$ и $w \neq s$, буквы слова $E_{z}$ суть 0 . Значит, $\delta_{z} \in r\left(\varepsilon_{w}\right)$. Поскольку $\delta_{z} \notin \Theta$, имеем $\delta_{z} \in r\left(\iota_{w}\right)$. Следовательно, $\delta_{z} \in \bigcup_{j \in M} r\left(\iota_{j}\right)$, где $M=\{1, \ldots, n+1\} \backslash\{p, s\}$. В результате получаем, что $\left(\left\{\iota_{1}, \ldots, \iota_{n+1}\right\} \backslash\left\{\iota_{p}, \iota_{s}\right\}\right) \supset \iota_{p}=\delta_{v}$. Так как элементы $\iota_{1}, \ldots, \iota_{n+1}$ выражены через $\varepsilon_{1}, \ldots, \varepsilon_{n+1}$, то и элемент $\delta_{v}$ выражен через $\varepsilon_{1}, \ldots, \varepsilon_{n+1}$.

Среди $E_{1}, \ldots, E_{l}$ имеются $(n+1) n / 2$ слов, в которые буква 0 входит ровно два раза. Тогда еще $(n+1) n / 2$ элемент множества $\left\{\delta_{1}, \ldots, \delta_{l}\right\}$ выразили через $\varepsilon_{1}, \ldots, \varepsilon_{n+1}$. Повторив $n$ раз такой процесс, элементы $\delta_{1}, \ldots, \delta_{l}$ выразим через $\varepsilon_{1}, \ldots, \varepsilon_{n+1}$. Лемма доказана.

Лемма 7. Если $k \geqslant 4,1 \leqslant m \leqslant n, l=2^{n+1}-2, \quad 1 \leqslant s_{1}<\cdots<s_{m} \leqslant n+1$, a $\tau_{i}$ есть $\varepsilon_{s_{i}}$ или $\neg \varepsilon_{s_{i}}$, әде $1 \leqslant i \leqslant m$, то в псевдобулевой алгебре $\Omega_{k}^{l}$ вернь соотношения $r\left(\tau_{1} \vee \ldots \vee \tau_{m}\right) \subseteq\left\{\alpha, \delta_{1}, \ldots, \delta_{l}\right\}$ u $r\left(\tau_{1} \vee \ldots \vee \tau_{m}\right) \backslash\{\alpha\} \neq \varnothing$. 
ДоКАЗАТЕЛЬСТВо. Пусть выполнены условия леммы. Тогда согласно построению псевдобулевой алгебры $\Omega_{k}^{l}$ и определению элементов $\varepsilon_{1}, \ldots, \varepsilon_{n+1}$ имеем $\mathfrak{M}\left(I\left(\Omega_{k}^{l}\right)\right)=\left\{\alpha, \delta_{1}, \ldots, \delta_{l}\right\}$ и $r\left(\varepsilon_{i}\right) \subseteq\left\{\delta_{1}, \ldots, \delta_{l}\right\}$, где $1 \leqslant i \leqslant n+1$ (см. рис. $\left.12-14\right)$. Поэтому с помошью леммы 5 из [17] получаем, что $r\left(\neg \varepsilon_{i}\right)=\left\{\alpha, \delta_{1}, \ldots, \delta_{l}\right\} \backslash r\left(\varepsilon_{i}\right)$, где $1 \leqslant i \leqslant n+1$. Так как $l \geqslant 2$, то для любых $i$ и $j$, удовлетворяющих условиям $1 \leqslant i \leqslant l, 1 \leqslant j \leqslant l$ и $i \neq j$, в $\Omega_{4}^{l}$ верно равенство $\delta_{i} \vee \delta_{j}=\varphi$, а в $\Omega_{k}^{l}$, где $k \geqslant 5,-$ равенство $\delta_{i} \vee \delta_{j}=\varphi \& \varkappa$ (см. рис. 12-14). Кроме того, для всякого $i$, удовлетворяющего условию $1 \leqslant i \leqslant l$, в $\Omega_{4}^{l}$ верно равенство $\delta_{i} \vee \alpha=\omega$, в $\Omega_{5}^{l}-$ равенство $\delta_{i} \vee \alpha=\varkappa$, а в $\Omega_{k}^{l}$, где $k \geqslant 6,-$ равенство $\delta_{i} \vee \alpha=\lambda \& \varkappa$ (см. рис. 12-14).

Пусть $\zeta_{1} \in r\left(\tau_{1}\right), \ldots, \zeta_{m} \in r\left(\tau_{m}\right)$. Если $\zeta_{1}=\cdots=\zeta_{m}$, то в $\Omega_{k}^{l}$ верно равенство $\zeta_{1} \vee \ldots \vee \zeta_{m}=\zeta_{1}$. Пусть среди $\zeta_{1}, \ldots, \zeta_{m}$ найдутся хотя бы два различных элемента. Если $\zeta_{1} \neq \alpha, \ldots, \zeta_{m} \neq \alpha$, то в $\Omega_{4}^{l}$ верно равенство $\zeta_{1} \vee \ldots \vee \zeta_{m}=\varphi$, а в $\Omega_{k}^{l}$, где $k \geqslant 5,-$ равенство $\zeta_{1} \vee \ldots \vee \zeta_{m}=\varphi \& \varkappa$. Если среди $\zeta_{1}, \ldots, \zeta_{m}$ сушествует элемент, равньй $\alpha$, то в $\Omega_{4}^{l}$ верно равенство $\zeta_{1} \vee \ldots \vee \zeta_{m}=\omega$, в $\Omega_{5}^{l}-$ равенство $\zeta_{1} \vee \ldots \vee \zeta_{m}=\varkappa$, а в $\Omega_{k}^{l}$, где $k \geqslant 6,-$ равенство $\zeta_{1} \vee \ldots \vee \zeta_{m}=\lambda \& \varkappa$.

Множество $\left\{\zeta_{1} \vee \ldots \vee \zeta_{m} \mid \zeta_{1} \in r\left(\tau_{1}\right), \ldots, \zeta_{m} \in r\left(\tau_{m}\right)\right\}$ обозначим буквой $\Xi$. Так как $1 \leqslant m \leqslant n$, то среди $E_{1}, \ldots, E_{l}$ существует такое слово $E_{z}$, что его $s_{i \text {-я }}$ буква, где $1 \leqslant i \leqslant m$, есть 0 , если $\tau_{i}=\varepsilon_{s_{i}}$, и есть 1 , если $\tau_{i}=\neg \varepsilon_{s_{i}}$. Если $\tau_{i}=\varepsilon_{s_{i}}$, где $1 \leqslant i \leqslant m$, то имеем $\delta_{z} \in r\left(\tau_{i}\right)$. Если $\tau_{i}=\neg \varepsilon_{s_{i}}$, где $1 \leqslant i \leqslant m$, то $\delta_{z} \notin r\left(\varepsilon_{s_{i}}\right)$ и $r\left(\tau_{i}\right)=\left\{\alpha, \delta_{1}, \ldots, \delta_{l}\right\} \backslash r\left(\varepsilon_{s_{i}}\right)$, а поэтому имеем $\delta_{z} \in r\left(\tau_{i}\right)$. Тогда во множестве $\Xi$ найдется элемент, равный $\delta_{z}$. Легко видеть, что $\tau_{1} \vee \ldots \vee \tau_{m}=\bar{\Xi}$. Кроме того, в $\Omega_{4}^{l}$ верно неравенство $\delta_{z} \leqslant \varphi$, в $\Omega_{5}^{l}$ - неравенства $\delta_{z} \leqslant \varphi$ и $\delta_{z} \leqslant \varkappa$, а в $\Omega_{k}^{l}$, где $k \geqslant 6,-$ неравенства $\delta_{z} \leqslant \varphi, \delta_{z} \leqslant \varkappa$ и $\delta_{z} \leqslant \lambda$. Следовательно, $r\left(\tau_{1} \vee \ldots \vee \tau_{m}\right) \subseteq\left\{\alpha, \delta_{1}, \ldots, \delta_{l}\right\}$ и $r\left(\tau_{1} \vee \ldots \vee \tau_{m}\right) \backslash\{\alpha\} \neq \varnothing$. Лемма доказана.

Двухэлементную булеву алгебру, задающую классическую пропозициональную логику и являюшуюся также псевдобулевой алгеброй, обозначим через $\Lambda$. Для определенности и удобства изложения материала будем считать, что $\Lambda=$ $\{0,1\}$ и 1 - выделенный элемент этой алгебры.

Пусть $n \geqslant 1, b_{1}, \ldots, b_{n}$ - различные пропозициональные переменные и $f$ - оценка переменных $b_{1}, \ldots, b_{n}$ в $\Lambda$. Если $f\left(b_{i}\right)=1$, то положим $V_{i}^{f}=\neg b_{i}$ и $W_{i}^{f}=b_{i}$. Если $f\left(b_{i}\right)=0$, то положим $V_{i}^{f}=b_{i}$ и $W_{i}^{f}=\neg b_{i}$. Для $f$ построим элементарные дизъюнкцию $D_{f}^{n}$ и конъюнкцию $K_{f}^{n}$, положив $D_{f}^{n}=V_{1}^{f} \vee \ldots \vee V_{n}^{f}$ и $K_{f}^{n}=W_{1}^{f} \& \ldots \& W_{n}^{f}$.

Легко видеть, что число всех различных оценок переменных $b_{1}, \ldots, b_{n}$ в $\Lambda$ равно $2^{n}$. Пусть это будут $f_{1}, \ldots, f_{2^{n}}$. Множества формул $\left\{K_{f_{i}}^{n} \mid 1 \leqslant i \leqslant 2^{n}\right\}$ и $\left\{D_{f_{i}}^{n} \vee D_{f_{j}}^{n} \mid 1 \leqslant i \leqslant 2^{n}, 1 \leqslant j \leqslant 2^{n}\right\}$ обозначим через $\mathfrak{S}_{n}$ и $\mathfrak{F}_{n}$ соответственно.

В дальнейшем совершенную дизъюнктивную нормальную форму пропозициональной формулы $X$ (см. [2, с. 124]) будем обозначать через $D_{X}$. Если $X$ - тождественно ложная формула, то $D_{X}=b \& \neg b$, где $b$ - какая-нибудь переменная формулы $X$.

ЛЕмма 8. Какова бы ни была пропозициональная формула $Y$, построенная из переменных $b_{1}, \ldots, b_{n}$, существует такое множество формул $\mathfrak{Y}$, ито $\mathfrak{Y} \subseteq \mathfrak{F}_{n}, \quad \mathfrak{Y} \neq \varnothing, \vdash \overline{\mathfrak{Y}} \supset Y u \vdash \neg \neg \overline{\mathfrak{Y}} \equiv \neg \neg Y$.

ДоКАЗАТЕЛЬСТво. Пусть $Y$ не является тождественно ложной формулой. Тогда существует такое множество формул $\mathfrak{X}$, что $\mathfrak{X} \subseteq \mathfrak{S}_{n}, \mathfrak{X} \neq \varnothing$ и $D_{Y}=\bigvee_{X \in \mathfrak{X}} X$. 
С помошью законов дистрибутивности получаем, что $\vdash D_{Y} \equiv\left(\&_{i=1}^{m} Z_{i}\right)$, где $Z_{i}=B_{i_{1}} \vee \ldots \vee B_{i_{k}}, 1 \leqslant i_{1}<\cdots<i_{k} \leqslant n$, а $B_{s}=b_{s}$, или $B_{s}=\neg b_{s}$, или $B_{s}=b_{s} \vee \neg b_{s}$ для $s \in\left\{i_{1}, \ldots, i_{k}\right\}$. Если $Y$ - тождественно ложная формула, то $D_{Y}=b_{1} \& \neg b_{1}$, и поэтому $D_{Y}$ эквивалентна в $\mathbf{H}$ формуле указанного в предыдущем случае вида (т. е. эквивалентна сама себе).

Рассмотрим формулу $Z_{i}$, где $1 \leqslant i \leqslant m$. Для $j$, удовлетворяюшего условию $1 \leqslant j \leqslant n$, положим $C_{j}=B_{j}$, если $j \in\left\{i_{1}, \ldots, i_{k}\right\}$, и $C_{j}=b_{j} \& \neg b_{j}$, если $j \notin\left\{i_{1}, \ldots, i_{k}\right\}$. Положим $V_{i}=C_{1} \vee \ldots \vee C_{n}$. Легко видеть, что $\vdash Z_{i} \supset V_{i}$. Поскольку $\vdash C_{j} \supset Z_{i}$, где $1 \leqslant j \leqslant n$, имеем $\vdash V_{i} \supset Z_{i}$. Следовательно, $\vdash Z_{i} \equiv V_{i}$. С помошью законов дистрибутивности получаем, что $\vdash V_{i} \equiv\left(T_{1} \& \ldots \& T_{q}\right)$, где $T_{l}=W_{1} \vee \ldots \vee W_{n}$, а $W_{t}=b_{t}$, или $W_{t}=\neg b_{t}$, или $W_{t}=b_{t} \vee \neg b_{t}$. Легко видеть, что для $T_{l}$ в $\mathfrak{F}_{n}$ сушествует такая формула $R$, что $\vdash T_{l} \equiv R$. Следовательно, для $D_{Y}$ сушествует такое множество формул $\mathfrak{Y}$, что $\mathfrak{Y} \subseteq \mathfrak{F}_{n}, \mathfrak{Y} \neq \varnothing$ и $\vdash D_{Y} \equiv \overline{\mathfrak{Y}}$.

В силу [2, лемма 13$]$ имеем $\vdash D_{Y} \supset Y$. Так как $D_{Y} \equiv Y$ - тождественно истинная формула, то $\vdash \neg \neg\left(D_{Y} \equiv Y\right)$, и поэтому имеем $\vdash \neg \neg D_{Y} \equiv \neg \neg Y$. Следовательно, $\vdash \overline{\mathfrak{Y}} \supset Y$ и $\vdash \neg \neg \overline{\mathfrak{Y}} \equiv \neg \neg Y$. Лемма доказана.

Пропозициональную формулу $\neg \neg b \vee(\neg \neg b \supset b)$ обозначим через $Q$, а множество формул $\left\{\neg \neg X \vee((\neg \neg X \& \neg \neg Y) \supset Y) \mid X, Y \in \mathfrak{F}_{n}\right\}$ - через $\mathfrak{Q}_{n}$. Отметим, что формулы множества $\mathfrak{Q}_{n}$ построены из переменных $b_{1}, \ldots, b_{n}$.

Лемма 9. Если $k \geqslant 4, n \geqslant 1 u l=2^{n+1}-2$, то каждая формула множества $\mathfrak{Q}_{n}$ общезначима во множестве $\left\{\varepsilon_{1}, \ldots, \varepsilon_{n+1}\right\}$ псевдобулевой алгебры $\Omega_{k}^{l}$.

ДокаЗАТЕЛЬСтво. Пусть $Z \in \mathfrak{Q}_{n}$. Тогда $Z=\neg \neg X \vee((\neg \neg X \& \neg \neg Y) \supset Y)$, где $X, Y \in \mathfrak{F}_{n}$. Поэтому формулы $X, Y$ и $Z$ построены из переменных $b_{1}, \ldots, b_{n}$. Пусть $f$ - оценка переменных $b_{1}, \ldots, b_{n}$ элементами множества $\left\{\varepsilon_{1}, \ldots\right.$ $\left.\ldots, \varepsilon_{n+1}\right\}$ алгебры $\Omega_{k}^{l}$. Так как $X \in \mathfrak{F}_{n}$, то имеем $f(X)=\tau_{1} \vee \ldots \vee \tau_{m}$, где $1 \leqslant m \leqslant n, 1 \leqslant s_{1}<\cdots<s_{m} \leqslant n+1$, а $\tau_{i}$ есть $\varepsilon_{s_{i}}$, или $\neg \varepsilon_{s_{i}}$, или $\varepsilon_{s_{i}} \vee \neg \varepsilon_{s_{i}}$. По тем же соображениям имеем $f(Y)=\sigma_{1} \vee \ldots \vee \sigma_{t}$, где $1 \leqslant t \leqslant n$, $1 \leqslant q_{1}<\cdots<q_{t} \leqslant n+1$, а $\sigma_{j}$ есть $\varepsilon_{q_{j}}$, или $\neg \varepsilon_{q_{j}}$, или $\varepsilon_{q_{j}} \vee \neg \varepsilon_{q_{j}}$.

Пусть существует такое $u$, что $1 \leqslant u \leqslant m$ и $\tau_{u}=\varepsilon_{s_{u}} \vee \neg \varepsilon_{s_{u}}$. Поскольку $\tau_{u} \leqslant f(X)$, имеем $\neg \neg \tau_{u} \leqslant \neg \neg f(X)$. Так как $\neg \neg \tau_{u}=\mathbf{1}$, то в $\Omega_{k}^{l}$ верно равенство $\neg \neg f(X)=\mathbf{1}$. Следовательно, $f(Z)=\mathbf{1}$.

Пусть для всякого $j$, удовлетворяющего условию $1 \leqslant j \leqslant t, \sigma_{j}$ есть $\varepsilon_{q_{j}}$ или $\neg \varepsilon_{q_{j}}$. Тогда по лемме 7 получаем, что $r(f(Y)) \subseteq\left\{\alpha, \delta_{1}, \ldots, \delta_{l}\right\}$. Согласно построению псевдобулевой алгебры $\Omega_{k}^{l}$ имеем $\mathfrak{M}\left(I\left(\Omega_{k}^{l}\right)\right)=\left\{\alpha, \delta_{1}, \ldots, \delta_{l}\right\}$ (см. рис. $12-14)$. В силу $[17$, лемма 5,3$)]$ для любого $\xi$ из $\mathfrak{M}\left(I\left(\Omega_{k}^{l}\right)\right)$ в $\Omega_{k}^{l}$ верно равенство $\neg \neg \xi=\xi$. Поэтому имеем

$$
\begin{aligned}
\neg \neg f(Y) & =\neg \neg \overline{\{\zeta \mid \zeta \in r(f(Y))\}}=\overline{\{\neg \neg \zeta \mid \zeta \in r(f(Y))\}} \\
& =\overline{\{\zeta \mid \zeta \in r(f(Y))\}}=f(Y) .
\end{aligned}
$$

Следовательно, $f(Z)=\mathbf{1}$.

Пусть для всякого $i$, удовлетворяюшего условию $1 \leqslant i \leqslant m, \tau_{i}$ есть $\varepsilon_{s_{i}}$ или $\neg \varepsilon_{s_{i}}$. Пусть сушествует такое $w$, что $1 \leqslant w \leqslant t$ и $\sigma_{w}=\varepsilon_{s_{w}} \vee \neg \varepsilon_{s_{w}}$. Тогда по лемме 7 имеем

$$
r(f(X)) \subseteq\left\{\alpha, \delta_{1}, \ldots, \delta_{l}\right\}, \quad r(f(X)) \backslash\{\alpha\} \neq \varnothing .
$$


Как и вьше, получаем, что $\neg \neg f(X)=f(X)$ и $\neg \neg f(Y)=\mathbf{1}$. Поэтому в $\Omega_{4}^{l}$ верно неравенство $\varphi \leqslant f(Y)$ и в $\Omega_{k}^{l}$, где $k \geqslant 5,-$ неравенство $\varphi \& \varkappa \leqslant f(Y)$ (см. рис. 12-14). Кроме того, для любого $j$, удовлетворяющего условию $1 \leqslant j \leqslant l$, в $\Omega_{4}^{l}$ верно неравенство $\delta_{j} \leqslant \varphi$, а в $\Omega_{k}^{l}$, где $k \geqslant 5$, - неравенство $\delta_{j} \leqslant \varphi \& \varkappa$. Поскольку $r(f(X)) \subseteq\left\{\alpha, \delta_{1}, \ldots, \delta_{l}\right\}$ и $r(f(X)) \backslash\{\alpha\} \neq \varnothing$, имеем $f(X) \supset f(Y)=1$. Следовательно, $f(Z)=\mathbf{1}$. Лемма доказана.

Напомним, что $Q=\neg \neg b \vee(\neg \neg b \supset b)$, а формулы множества $\mathfrak{Q}_{n}$ построены из переменных $b_{1}, \ldots, b_{n}$.

ТЕОРема 4. Пусть $n \geqslant 1$. Тогда верны следующие утверждения:

1) исчисление $\mathbf{H}+\mathfrak{Q}_{n}$ является аксиоматизацией логики $\mathfrak{L}(Q)$;

2) если пропозициональная формула $S$ построена из переменных $b_{1}, \ldots, b_{n}$ и принадлежит логике $\mathfrak{L}(Q)$, mо $\vdash \overline{\mathfrak{Q}_{n}} \supset S$.

ДоКАЗАТЕЛЬСТво. Пусть $n \geqslant 1$. Докажем утверждение 1$)$. Для этого достаточно показать, что $\overline{\mathfrak{Q}_{n}} \vdash Q$ и $Q \vdash \overline{\mathfrak{Q}_{n}}$. Согласно построению множества $\mathfrak{Q}_{n}$ формула $\neg \neg V \vee((\neg \neg V \& \neg \neg W) \supset W)$, где $V=W=\left(b_{1} \vee \ldots \vee b_{n}\right) \vee\left(b_{1} \vee \ldots \vee b_{n}\right)$, принадлежит $\mathfrak{Q}_{n}$. Подставив в нее вместо переменных $b_{1}, \ldots, b_{n}$ переменную $b$, получим формулу, эквивалентную в $\mathbf{H}$ формуле $Q$. Следовательно, $\overline{\mathfrak{Q}_{n}} \vdash Q$.

Пусть $X, Y \in \mathfrak{F}_{n}$. Легко видеть, что $Q \vdash \neg \neg(X \& Y) \vee(\neg \neg(X \& Y) \supset(X \& Y))$. Кроме того, в Н выводимы эквивалентности

$$
\begin{aligned}
& (\neg \neg(X \& Y) \vee(\neg \neg(X \& Y) \supset(X \& Y))) \\
& \quad \equiv((\neg \neg X \& \neg \neg Y) \vee(((\neg \neg X \& \neg \neg Y) \supset X) \&((\neg \neg X \& \neg \neg Y) \supset Y))) \\
& \quad \equiv((\neg \neg X \vee((\neg \neg X \& \neg \neg Y) \supset X)) \&(\neg \neg X \vee((\neg \neg X \& \neg \neg Y) \supset Y)) \\
& \quad \&(\neg \neg Y \vee((\neg \neg X \& \neg \neg Y) \supset X)) \&(\neg \neg Y \vee((\neg \neg X \& \neg \neg Y) \supset Y))) .
\end{aligned}
$$

Значит, $Q \vdash \neg \neg X \vee((\neg \neg X \& \neg \neg Y) \supset Y)$. Следовательно, $Q \vdash \overline{\mathfrak{Q}_{n}}$.

Докажем утверждение 2). Пусть пропозициональная формула $S$ построена из переменных $b_{1}, \ldots, b_{n}$ и принадлежит логике $\mathfrak{L}(Q)$. Тогда сушествует такое конечное множество пропозициональных формул $\mathfrak{Z}$, что $\mathfrak{Z} \neq \varnothing$,

$$
\vdash \overline{\{\neg \neg Z \vee(\neg \neg Z \supset Z) \mid Z \in \mathfrak{Z}\}} \supset S
$$

и каждая формула из $\mathfrak{Z}$ построена из переменных $b_{1}, \ldots, b_{n}$. Для нашей цели достаточно показать, что $\vdash \overline{\mathfrak{Q}_{n}} \supset(\neg \neg Z \vee(\neg \neg Z \supset Z))$, где $Z \in \mathfrak{Z}$. В самом деле, пусть $Z \in \mathfrak{Z}$. Тогда по лемме 8 для $Z$ сушествует такое множество формул $\mathfrak{Y}$, что $\mathfrak{Y} \subseteq \mathfrak{F}_{n}$, $\mathfrak{Y} \neq \varnothing, \vdash \overline{\mathfrak{Y}} \supset Z$ и $\vdash \neg \neg \overline{\mathfrak{Y}} \equiv \neg \neg Z$.

Пусть $X, Y \in \mathfrak{Y}$. Легко видеть, что $\vdash \overline{\mathfrak{Q}_{n}} \supset(\neg \neg X \vee((\neg \neg X \& \neg \neg Y) \supset Y))$. Поскольку $\vdash((\neg \neg X \& \neg \neg Y) \supset Y) \supset(\overline{\{\neg \neg V \mid V \in \mathfrak{Y}\}} \supset Y)$, имеем

$$
\vdash(\neg \neg X \vee((\neg \neg X \& \neg \neg Y) \supset Y)) \supset(\neg \neg X \vee(\overline{\{\neg \neg V \mid V \in \mathfrak{Y}\}} \supset Y)) .
$$

Значит, $\vdash \overline{\mathfrak{Q}_{n}} \supset\{\overline{\neg \neg X \vee(\{\overline{\neg \neg V \mid V \in \mathfrak{Y}}\} \supset Y) \mid X, Y \in \mathfrak{Y}}\}$. Нетрудно проверить, что в $\mathbf{H}$ выводимы следуюшие эквивалентности:

$$
\begin{aligned}
& (\neg \neg \overline{\mathfrak{Y}} \vee(\neg \neg \overline{\mathfrak{Y}} \supset \overline{\mathfrak{Y}})) \equiv(\overline{\{\neg \neg V \mid V \in \mathfrak{Y}\}} \vee(\overline{\{\neg \neg V \mid V \in \mathfrak{Y}\}} \supset \overline{\mathfrak{Y}})) \\
& \equiv(\overline{\{\neg \neg V \mid V \in \mathfrak{Y}\}} \vee \overline{\{\overline{\neg \neg V \mid V \in \mathfrak{Y}}\} \supset Y \mid Y \in \mathfrak{Y}\}}) \\
& \equiv \overline{\{\neg \neg X \vee(\overline{\{\neg \neg V \mid V \in \mathfrak{Y}\}} \supset Y) \mid X, Y \in \mathfrak{Y}\}} \text {. }
\end{aligned}
$$


Следовательно, $\vdash \overline{\mathfrak{Q}_{n}} \supset(\neg \neg \overline{\mathfrak{Y}} \vee(\neg \neg \overline{\mathfrak{Y}} \supset \overline{\mathfrak{Y}}))$. Так как $\vdash \overline{\mathfrak{Y}} \supset Z$ и $\vdash \neg \neg \overline{\mathfrak{Y}} \equiv \neg \neg Z$, то имеем $\vdash \overline{\mathfrak{Q}_{n}} \supset(\neg \neg Z \vee(\neg \neg Z \supset Z))$. Теорема доказана.

Теорема 5 [9, теорема 3]. Если пропозичиональная формула $Y$ принадлежит логике $\mathfrak{L}(Q)$ и опровержима в псевдобулевой алгебре $\Omega$, то логика $\mathfrak{L}(Y)$ не обладает свойством простой подстановки.

ДоКАЗАТЕЛЬСТвО. Пусть формула $Y$ удовлетворяет условию теоремы. Предположим, что логика $\mathfrak{L}(Y)$ обладает свойством простой подстановки. Тогда существует такая пропозициональная формула $T$, что исчисление $\mathbf{H}+\{T\}$ является аксиоматизацией логики $\mathfrak{L}(Y)$ и обладает этим свойством. Поэтому $T \in \mathfrak{L}(Q)$ и $T$ опровержима в псевдобулевой алгебре $\Omega$. Пусть формула $T$ построена из переменных $d_{1}, \ldots, d_{n}$. Построим формулу $S$, заменив в $T$ переменные $d_{1}, \ldots, d_{n}$ на переменные $b_{1}, \ldots, b_{n}$ соответственно. Легко видеть, что $S \in \mathfrak{L}(Q), S$ опровержима в $\Omega$ и исчисление $\mathbf{H}+\{S\}$ обладает свойством простой подстановки. Тогда существует такое $k$, что $k \geqslant 4$ и $S$ опровержима в псевдобулевой алгебре $\Omega_{2 k}$. В силу утверждения 1$)$ леммы 6 алгебра $\Omega_{2 k}$ вложима в псевдобулеву алгебру $\Omega_{k}^{l}$, где $l=2^{n+1}-2$. Тогда $S$ опровержима в $\Omega_{k}^{l}$.

По теореме 1 формула $S$ наследственно общезначима в $\Omega_{k}^{l}$. Так как $S \in \mathfrak{L}(Q)$ и $S$ построена из переменных $b_{1}, \ldots, b_{n}$, то в силу теоремы 4,2$)$ имеем $\vdash \overline{\mathfrak{Q}_{n}} \supset S$. Тогда формула $\overline{\mathfrak{Q}_{n}} \supset S$ общезначима в $\Omega_{k}^{l}$. По лемме 9 каждая формула множества $\mathfrak{Q}_{n}$ общезначима во множестве $\left\{\varepsilon_{1}, \ldots, \varepsilon_{n+1}\right\}$ алгебры $\Omega_{k}^{l}$. Значит, $\overline{\mathfrak{Q}_{n}}$ и, следовательно, формула $S$ обладают этим же свойством. В силу утверждения 3$)$ леммы 6 элементы $\varepsilon_{1}, \ldots, \varepsilon_{n+1}$ являются образуюшими алгебры $\Omega_{k}^{l}$. Тогда $S$ обшезначима в $\Omega_{k}^{l}$. Противоречие. Следовательно, логика $\mathfrak{L}(Y)$ не обладает свойством простой подстановки. Теорема доказана.

Согласно результатам работы [6] формула $Q$ опровержима в псевдобулевой алгебре $\Omega$. Поэтому из теорем 4,5 вытекает такое следствие.

СЛЕДСТВИЕ 1. Исчисление $\mathbf{H}+\mathfrak{Q}_{n}$ не обладает свойством простой подстановки.

Выясним, какие из логик, порожденных пропозициональными формулами от одной переменной $b$, обладают свойством простой подстановки. Так как каждая такая формула эквивалентна в Н некоторой формуле из множества $\left\{b \supset b, P_{0}, P_{1}, \ldots\right\}$ [6], то для нашей цели достаточно рассмотреть только логики $\mathfrak{L}\left(P_{0}\right), \mathfrak{L}\left(P_{1}\right), \ldots$ Нетрудно проверить, что $\mathfrak{L}\left(P_{0}\right)=\mathfrak{L}\left(P_{1}\right)=\mathfrak{L}\left(P_{2}\right)=\mathfrak{L}\left(P_{4}\right), \mathfrak{L}\left(P_{3}\right)=\mathfrak{L}\left(P_{6}\right)$ и $\mathfrak{L}\left(P_{5}\right)=\mathfrak{L}\left(P_{8}\right)$. Известно [1], что логики $\mathfrak{L}\left(P_{0}\right), \mathfrak{L}\left(P_{3}\right)$ и $\mathfrak{L}\left(P_{5}\right)$ обладают этим свойством. Согласно результатам работы [6] формула $P_{i}$, где $i=7$ или $i \geqslant 9$, принадлежит логике $\mathfrak{L}(Q)$ и опровержима в псевдобулевой алгебре $\Omega$. Поэтому из теоремы 5 вытекает такое следствие.

СЛЕДСТВИЕ $2\left[9\right.$, следствие 1]. Логика $\mathfrak{L}\left(P_{i}\right)$, əде $i=7$ или $i \geqslant 9$, не обладает свойством простой подстановки.

Это следствие обобшает результат из [1] о том, что аксиоматизация $\mathbf{H}+\left\{P_{i}\right\}$, где $i=7$ или $i \geqslant 9$, логики $\mathfrak{L}\left(P_{i}\right)$ не обладает свойством простой подстановки. 


\section{§5. О связи свойств отделимости и простой подстановки}

В этом параграфе докажем анонсированные в работе [9] результаты, устанавливающие связь между свойствами отделимости и простой подстановки.

Логики $\mathfrak{L}((b \supset d) \vee(d \supset b)), \mathfrak{L}(b \vee \neg b)$ и $\mathfrak{L}(\neg b \vee \neg \neg b)$ отделимы [20], [13]-[15] и обладают свойством простой подстановки [1]. Предтабличные логики

$$
\mathfrak{L}((\neg a \vee \neg \neg a) \&(b \vee(b \supset(c \vee(c \supset(d \vee \neg d)))))), \quad \mathfrak{L}(b \vee(b \supset(d \vee \neg d)))
$$

отделимы [15]-[17], но не обладают свойством простой подстановки [5]. Логика $\mathfrak{L}\left(P_{i}\right)$, где $i \geqslant 10$, не отделима [13], [15] и согласно следствию 2 не обладает свойством простой подстановки. Табличные логики обладают свойством простой подстановки [4], но среди них имеются не нормализуемые и, следовательно, не отделимые логики [13], [15]. Поэтому возникает вопрос: каким образом связаны между собой отделимые и обладающие свойством простой подстановки аксиоматизации суперинтуиционистских пропозициональных логик? В частности, естественно спросить: существует ли такая логика, что некоторая ее аксиоматизация обладает этими двумя свойствами? Следующие результаты частично дают ответы на эти вопросы.

ТЕОРема 6 [9, теорема 4]. Если суперинтуиционистская пропозициональная логика отлична от абсолютно противоречивой и содержит формулу от одной переменной, не выводимую в $\mathbf{H}$, то кажсдая ее нормальная аксиоматизачия не обладает свойством простой подстановки, а каждая ее аксиоматизация, обладающая свойством простой подстановки, не является нормальной.

ДокАЗАТЕЛЬство. Пусть суперинтуиционистская пропозициональная логика $L$ отлична от абсолютно противоречивой и содержит формулу $S$ от одной переменной $d$, не выводимую в $\mathbf{H}$. Пусть $\mathbf{H}+\mathfrak{X}$ - нормальная аксиоматизация логики $L$. Тогда $\mathfrak{X} \neq \varnothing$ и каждая формула из $\mathfrak{X}$ является $\supset \vee \&$-формулой или $\supset \neg$-формулой. Пусть формулы множества $\mathfrak{X}$ построены из переменных $b_{1}, \ldots, b_{n}$.

Пусть $X \in \mathfrak{X}$. Если $X-\supset \vee \&$-формула, то индукцией по построению формулы $X$ получаем, что $\mathbf{F}\left(b_{1}, \ldots, b_{n}|d, \ldots, d| X\right)$ эквивалентна в $\mathbf{H}$ одной из формул $d$ или $d \supset d$. Если $X-\supset$-форомула, то аналогичным образом получаем, что $\mathbf{F}\left(b_{1}, \ldots, b_{n}|d, \ldots, d| X\right)$ эквивалентна в $\mathbf{H}$ одной из формул $\neg(d \supset d)$, или $d$, или $\neg d$, или $\neg \neg d$, или $\neg \neg d \supset d$, или $d \supset d$. Так как логика $L$ отлична от абсолютно противоречивой, то $\vdash \overline{\{Y[d] \mid Y \in \mathfrak{X}\}} \equiv(d \supset d)$ или $\vdash \overline{\{Y[d] \mid Y \in \mathfrak{X}\}} \equiv(\neg \neg d \supset d)$.

Предположим, что исчисление $\mathbf{H}+\mathfrak{X}$ обладает свойством простой подстановки. Предположим, что $\vdash \overline{\{Y[d] \mid Y \in \mathfrak{X}\}} \equiv(d \supset d)$. Так как $L$ содержит формулу $S$ от одной переменной $d$, то $\vdash\{Y[d] \mid Y \in \mathfrak{X}\} \supset S$, и поэтому имеем $\vdash S$, что противоречит условию теоремы. Следовательно, $\vdash\{Y[d] \mid Y \in \mathfrak{X}\} \equiv(\neg \neg d \supset d)$. Тогда $L-$ классическая логика. Значит, $d \vee \neg d \in L$. Тогда $\vdash \overline{\{Y[d] \mid Y \in \mathfrak{X}\}} \supset(d \vee \neg d)$, и поэтому имеем $\vdash(\neg \neg d \supset d) \supset(d \vee \neg d)$. Но эта формула не вьводима в Н. Противоречие. Следовательно, исчисление $\mathbf{H}+\mathfrak{X}$ не обладает свойством простой подстановки.

Пусть аксиоматизация $\mathbf{H}+\mathfrak{Y}$ логики $L$ обладает свойством простой подстановки. Если бы исчисление $\mathbf{H}+\mathfrak{Y}$ было нормальным, то в силу доказанного выше $\mathbf{H}+\mathfrak{Y}$ не обладало бы свойством простой подстановки, что приводило бы к противоречию. Значит, $\mathbf{H}+\mathfrak{Y}$ не является нормальным. Теорема доказана. 
Покажем, что табличные и предтабличные логики находятся в области действия теоремы 6.

Пусть $L$ - табличная логика, отличная от абсолютно противоречивой. Тогда в силу [7], [33] логика $L$ содержит формулу $B_{n}=\bigvee_{0 \leqslant i<j \leqslant n}\left(b_{i} \equiv b_{j}\right)$ при некотором $n$, где $n \geqslant 1$ и $b_{0}, \ldots, b_{n}$ - различные пропозициональные переменные. Согласно результатам работы [6] формулы $P_{2+4 i}$, где $0 \leqslant i \leqslant n$ и $n \geqslant 1$, удовлетворяют следуюшим условиям:

$$
\vdash P_{j} \supset P_{l}, \quad \vdash\left(P_{l} \supset P_{j}\right) \equiv P_{j}, \quad j, l \in\{2+4 i \mid 0 \leqslant i \leqslant n\}, \quad j<l .
$$

Поэтому имеем $\vdash \mathbf{F}\left(b_{i}(0 \leqslant i \leqslant n)\left|P_{2+4 i}\right| B_{n}\right) \equiv P_{2+4 n}$. Следовательно, $P_{2+4 n} \in L$ и $P_{2+4 n}$ не выводима в $\mathbf{H}$.

Известно [8], что предтабличных логик ровно три:

$$
\begin{aligned}
\mathfrak{L}((b \supset d) \vee(d \supset b)), \quad & \mathfrak{L}((\neg a \vee \neg \neg a) \&(b \vee(b \supset(c \vee(c \supset(d \vee \neg d)))))), \\
& \mathfrak{L}(b \vee(b \supset(d \vee \neg d))) .
\end{aligned}
$$

Нетрудно проверить, что первая и вторая содержат не выводимую в $\mathbf{H}$ формулу $\neg b \vee \neg \neg b$, а третья - не выводимую в $\mathbf{H}$ формулу $\neg \neg b \vee(\neg \neg b \supset b)$.

Легко видеть, что логика $\mathfrak{L}\left(P_{i}\right)$, где $i \geqslant 5$, находится в области действия теоремы 6. Поэтому из теоремы 6 вытекает такое следствие.

СледСТвиЕ 3 [9, следствие 2]. Пусть $L-$ табличная логика, отличная от абсолютно противоречивой, или предтабличная логика, или логика $\mathfrak{L}\left(P_{i}\right)$, где $i \geqslant 5$. Тогда каждая нормальная аксиоматизачия логики $L$ не обладает свойством простой подстановки, а каждая ее аксиоматизачия, обладающая свойством простой подстановки, не является нормальной.

\section{Список литературы}

1. Sasaki K. The simple substitution property of the intermediate propositional logics // Bulletin of the Section of Logic. Polish Acad. Sci. Inst. Phil. and Sociol. 1989. V. 18. № 3. P. 94-99.

2. Клини С. К. Введение в метаматематику. М.: ИЛ, 1957.

3. Wajsberg M. Untersuchungen über den Aussagenkalkül von A. Heyting // Wiadomos̀ci matematyczne. 1938. V. 46. P. 45-101.

4. Hosoi T., Sasaki K. Finite logics and simple substitution property // Bulletin of the Section of Logic. Polish Acad. Sci. Inst. Phil. and Sociol. 1990. V. 19. № 3. P. 74-78.

5. Sasaki K. The simple substitution property of the intermediate propositional logics of finite slices // Studia Logica. 1993. V. 52. № 1. P. 41-62.

6. Nishimura I. On formulas of one veriable in intuitionistic propositional calculus // J. Symbolic Logic. 1960. V. 25. № 4. P. 327-331.

7. Hosoi T. On intermediate logics. I // J. of the faculty of science. University Tokyo. Section I. 1967. V. 14. № 2. P. 293-312.

8. Максимова Л. Л. Предтабличные суперинтуиционистские логики // Алгебра и логика. 1972. T. 11. № 5. C. 558-570.

9. Хомич В.И. О свойстве простой подстановки для суперинтуиционистских пропозициональных логик // Докл. РАН. 2000. Т. 374. №3. С. 318-320.

10. Хомич В.И. О свойстве простой подстановки для суперинтуиционистских пропозициональных исчислений и логик // Tp. IV Международной конференции "Дискретные модели в теории управляющих систем". М.: МАКС Пресс, 2000. С. 125-127.

11. Kreisel G., Putnam H. Eine Unableitbarkeitsbeweismethode für den intuitionistischen Aussagenkalkül // Arch. f. Math. Logik Grundlagenforschung. 1957. V. 3. P. 74-78. 
12. McKay C. G. The non-separability of a certain finite extension of Heyting's propositional logic // Proceedings Koninklijke Nederlandse Akad. van Wetenschappen. Ser. A. 1968. V. 71. № 3. P. 312-315.

13. Hosoi T. Non-separable intermediate propositional logics // J. Tsuda College. 1976. V. 8. P. 13-18.

14. Хомич В. И. Теорема отделимости для суперинтуиционистских исчислений высказываний // ДАН СССР. 1976. Т. 229. №6. С. 1327-1329.

15. Хомич В.И. Отделимость суперинтуиционистских пропозициональных логик // Исследования по теории алгорифмов и математической логике. М.: Наука, 1979. С. 98-115.

16. Хомич В. И. О проблеме отделимости для суперинтуиционистских пропозициональных логик // ДАН СССР. 1980. Т. 254. №4. С. 820-823.

17. Хомич В.И. Об отделимых суперинтуиционистских пропозициональных исчислениях и о конъюнктивно неразложимых элементах в импликативных полуструктурах // Zeitschrift für mathematische Logik und Grundlagen der Mathematik. 1986. V. 32. № 2. P. $149-180$.

18. Хомич В. И. О свойствах суперинтуиционистских пропозициональных исчислений // Сиб. матем. журн. 1990. Т. 31. №6. С. 158-175.

19. Horn A. The separation theorem of intuitionist propositional calculus // J. Symbolic Logic. 1962. V. 27. № 4. P. 391-399.

20. Hosoi T. On the separation theorem of intermediate propositional calculi // Proc. Japan Acad. 1966. V. 42. № 6. P. 535-538.

21. Hosoi T. The separation theorem on the classical system // J. of the faculty of science. University Tokyo. Section I. 1966. V. 12. № 2. P. 223-230.

22. Карри Х. Б. Основания математической логики. М.: Мир, 1969.

23. Расева E., Сикорский Р. Математика метаматематики. М.: Наука, 1972.

24. Хомич В. И. О вложимости некоторых обобщений псевдобулевых алгебр // Докл. РАН. 1996. Т. 350. № 2. С. 174-177.

25. Хомич В.И. О вложении импликатур // Вопросы математической логики и теории алгоритмов. М.: ВЦ АН СССР, 1988. С. 17-33.

26. Биркгоф Г. Теория структур. М.: ИЛ, 1952.

27. Хомич В.И. О суперинтуиционистских пропозициональных логиках, связанных с частично упорядоченными множествами // Изв. АН СССР. Сер. матем. 1991. Т. 55. № 2. C. $384-406$.

28. Хомич В.И. О свойстве суперинтуиционистских пропозициональных исчислений, связанном с отделимостью этих исчислений // Математические вопросы кибернетики. Вып. 7. М.: Наука, 1998. С. 227-242.

29. Jaskows̀ki S. Recherches sur le système de la logique intuitioniste // Actualites scientifiques et industrielles. Paris. 1936. V. 393. P. 58-61.

30. Пильчаћ Б. Ю. Об исчислении задач // Укр. матем. журн. 1952. Т. 4. № 2. С. 174-194.

31. Хомич В.И. О представлении конечных псевдобулевых алгебр и об одном его применении // Матем. заметки. 1992. Т. 52. № 2. С. 127-137.

32. Хомич В.И. Об изоморфной вложимости псевдобулевых алгебр и некоторых их обобщений // Математические вопросы кибернетики. Вып. 8. М.: Наука, 1999. С. 191-218.

33. McKay C. G. On finite logics // Proceedings Koninklijke Nederlandse Akad. van Wetenschappen. Ser. A. 1967. V. 70. № 3. P. 363-365.

Вычислительный центр им. А. А. Дородницына РАН

Поступило в редакцию

19.VII.2001 